Article

\title{
Calcite Twinning in the Ordovician Martinsburg Formation, Delaware Water Gap, New Jersey, USA: Implications for Cleavage Formation and Tectonic Shortening in the Appalachian Piedmont Province
}

\author{
John P. Craddock ${ }^{1}{ }^{*}$, Maria Princen ${ }^{1}$, Jakob Wartman ${ }^{1}$, Haoran Xia ${ }^{2,3}$ and Junlai Liu ${ }^{2}$ \\ 1 Geology Department, Macalester College, St. Paul, MN 55105, USA; mariaprincen@gmail.com (M.P.); \\ jakob.wartman@gmail.com (J.W.) \\ 2 State Key Laboratory of Geological Processes and Mineral Resources, China University of Geosciences, \\ Haidian, Beijing 100083, China; haoranxi@usc.edu (H.X.); jliu@cugb.edu.cn (J.L.) \\ 3 Department of Geology, University of Southern California, Los Angeles, CA 90089-0740, USA \\ * Correspondence: craddock@macalester.edu; Tel.: +1-651-696-6620; Fax: +1-651-696-6122
}

Academic Editors: James Schmitt and Jesus Martinez-Frias

Received: 18 November 2015; Accepted: 5 February 2016; Published: 19 February 2016

\begin{abstract}
A traverse across the Stone Church syncline in the Ordovician Martinsburg turbidites reveals an axial planar cleavage $\left(\mathrm{N} 40^{\circ} \mathrm{E}\right.$, SE dips) in regional thrust-related folds $\left(\mathrm{N} 40^{\circ} \mathrm{E}\right.$, shallow plunges) and five phases of sparry calcite. Calcite fillings are bedding-parallel, cleavage-parallel, and one vein set cross-cuts both earlier phases; the youngest calcite filling is a bedding-parallel fault gouge that crosscuts the cleavage and preserves top-down-to-the-southeast normal fault kinematics. Calcite veins unique to disharmonically-folded calcareous siltstones (Maxwell, 1962) were also analyzed. Stable isotopic analysis $(\mathrm{O}, \mathrm{C})$ of all of the calcite phases indicates a uniform fluid source $\left(\delta^{13} \mathrm{C}\right.$ $-2.0, \delta^{18} \mathrm{O}-13.3 \mathrm{VPDB}$ ) and, potentially, a similar precipitation and mechanical twinning age. The twinning strains $\left(n=1341\right.$; average $\Delta \sigma=-32 \mathrm{MPa}$; average $\left.\varepsilon_{1}=-2.9 \%\right)$ in the calcite suite are consistent with SE-NW thrust shortening, and sub-horizontal shortening perpendicular to evolving axial planar cleavage planes in the Stone Church syncline. Calcareous siltstone layers within the Martinsburg Fm. turbidites share concordant bedding planes and are unique, chemically (XRF), but folded and cleaved differently than the surrounding clay-rich Martinsburg turbidites. Neither sediment type yielded detrital zircons. Electron backscatter X-ray diffraction (EBSD) and calcite twinning results in a folded calcareous siltstone layer preserving a layer-normal SE-NW shortening strain and Lattice Preferred Orientation (LPO). Shortening axes for the five-phase calcite suite trends $\sim \mathrm{N} 40^{\circ} \mathrm{W}$, consistent with tectonic transport associated with crystalline nappe emplacement of the Reading Prong within the Piedmont province.
\end{abstract}

Keywords: structural geology; Appalachian Piedmont; strain analysis

\section{Introduction}

Since Chapple [1] first unraveled the order and dynamics of shortening in a thin-skinned fold-and-thrust belt, using the Appalachian Mountains as a model, later workers have made enormous headway in understanding the deformational style in the Valley and Ridge (thin-skinned thrust belt) and foreland provinces. Recent studies in the Valley and Ridge and foreland provinces, which are separated by an oroclinal boundary, has generated controversy about the role of thrust belt translation and rotations relative to the adjacent, stable foreland [2,3]. There is no calcite twinning strain data from the internal, thick-skinned Piedmont province. The numerous calcite fillings (phases) in the folded 
and cleaved Ordovician Martinsburg Fm. in the Stone Church syncline provide a field site to evaluate the stress-strain relations that existed during the evolution of this fold and its axial planar cleavage and add the twinned calcite results to the existing literature on deformation and strain partitioning at this classic Piedmont province locale [4-7]. How does this strain fabric correlate with other indicators of tectonic shortening across the Appalachian orogenic belt and did the collisional orogeny involve significant thrust sheet rotation during shortening [8-10]?

Field relations suggest that the five-phases of calcite precipitated, and deformed plastically, while the host Ordovician Martinsburg Fm. was being folded and cleaved as the crystalline Taconic Reading Prong nappe moved to the northwest on the Martic thrust [11-16]. Sparry calcite is present as bedding-parallel veins, cleavage-parallel veins, and veins that cross-cut both. Also present are calcite veins unique to disharmonically-folded and cleaved calcareous siltstone layers (previously thought to be clastic dikes as the Martinsburg Fm. "dewatered"; see [4-7]) and we discovered a bedding-parallel, layered calcite fault zone with normal fault kinematics. We have compared the bulk geochemistry of the Martinsburg Fm. host and a calcareous siltstone (XRF), the stable isotope $(\mathrm{O}, \mathrm{C})$, and calcite twinning strain characteristics of each calcite phase in a section of folded Martinsburg Fm. turbidites exposed parallel to the Taconic orogeny thrust transport direction (SE-NW) along the Delaware River in New Jersey. Our goal was to determine if, (1) the many calcite vein phases hosted in the Martinsburg Fm. precipitated from a common fluid source and preserve twinning strains that are consistent with other observations of Taconic (or younger) shortening; (2) the relationship of the stress-strain field, as preserved by calcite precipitated parallel to the Martinsburg Fm. axial planar cleavage, is consistent with the results of Craddock et al. ([17]; horizontal shortening normal to the cleavage strike, horizontal extension parallel to strike) in the Gondwanide belt, and (3) the shortening strain fabrics in the Cambro-Ordovician sediments deformed in the thick-skinned Piedmont province of the Taconic orogeny remain parallel to the inferred thrust transport directions of younger (Acadian, Alleghenian) shortening events to the northwest in the Valley and Ridge and foreland provinces.

\section{Previous Work}

Structural studies in the Appalachian Mountains have revealed a variety of observations about deformation mechanisms and thrust belt mechanics, including finite strain studies by Cloos [18] and the role of pressure solution in the removal of rock materials during cleavage formation [19] in the Valley and Ridge province. Strain studies in the frontal Pine Mountain block record a layer-parallel shortening strain oriented parallel (NW-SE) to the inferred thrust transport direction and this fabric is parallel in the adjacent autochthonous footwall carbonates suggesting the thrust sheets moved without rotation [20-22]. Ong et al. [8] and Hnat and van der Pluijm [22] have documented the shortening strain parallelism between the thrust belt and foreland in additional Appalachian locations. Appalachian thrust belt deformation is also recorded in the foreland as salt-cored anticlines ( $200 \mathrm{~km}$ inboard from the thrust front; [23]), finite strains (200 km; [24,25]) and calcite twinning strains $(2000 \mathrm{~km}$; [26,27]) in the Paleozoic sediments of cratonic North America. The Delaware Water Gap exposures represent a rare traverse across the poorly exposed Piedmont province.

The internal (hinterland) Piedmont portion of the Appalachian Mountains is characterized by Cambrian (limestone)-Ordovician marine (Martinsburg Fm. flysch; 12,000 feet) sediments that were deformed by the late Ordovician Taconic orogeny. Geologic mapping by Drake et al. ([28,29] is the basis for the most recent local fieldguide prepared by Epstein ([30]; see geologic map and stratigraphic column) along the Delaware Water Gap. Folds trend $\sim \mathrm{N} 40^{\circ} \mathrm{E}$ with shallow plunges and are often underlain by SE-dipping thrusts. Minor folds, of many geometries, are observed and multiple, local cleavages are reported in the Martinsburg Fm. except along the Delaware Water gap section where only the axial-planar cleavge is observed. Grenville basement rocks, including marble inliers, were mobilized as thrust-cored nappes (Wilmington complex, Reading Prong) at 450 Ma deforming the Cambrian-Ordovician marine section along thrust faults that dip to the SE and transported these nappes to the NW [14,31,32]. These structures, including various Taconic klippe [33], were 
immediately buried by Taconic (450-443 Ma) molasse deposits, followed by deposition of the Silurian Shawangunk (Tuscarora sands)-Bloomsberg Fms. (redbeds) and Devonian Marcellus Shale [14] and constitute the hinterland Piedmont province of the Appalachians (Figure 1). The Delaware River valley exposes the Stone Church syncline (with minor folds), largely in the Martinsburg Fm. turbidites (Figure 1), which has revealed the interplay of folding [4], pressure solution volume loss due to cleavage formation [5-7,34], framboid-pressure shadow evolution in cleavage lithons [5,34], finite strain measurements [4,6,7], and AMS studies [35-37] toward developing the concept of strain partitioning in deformed, supracrustal sediments. Mechanical twinning in calcite is another piece of the strain history puzzle and there are numerous phases of sparry calcite veins to measure and interpret in this Piedmont structure relative to younger Acadian (Devonian) and Alleghenian (Permian) convergence and younger-still Triassic extension.

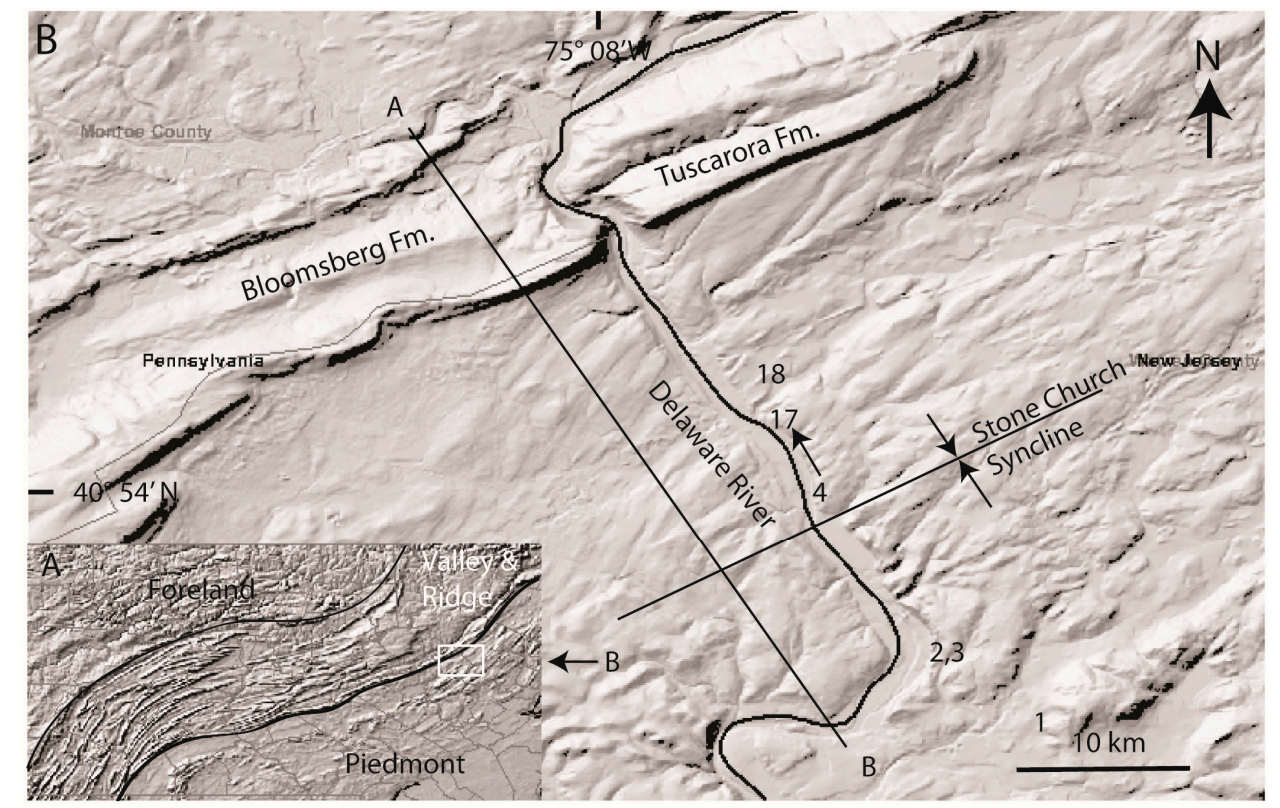

Figure 1. LIDAR base map of the Delaware Water Gap area (B) in New Jersey and Pennsylvania relative to the Appalachian Mountains (inset A with box for B). Sample sites are numbered. Cross section line (A-B) is from Maxwell [4] and in Figure 3; see also Herman et al. ([32], Figure 14 for regional subsurface structural relations).

\section{Methods}

\subsection{Geochemistry}

Stable isotopes were analyzed at the University of Michigan stable isotope laboratory. Drilled carbonate powder samples weighing a minimum of 10 micrograms were placed in stainless steel boats. Samples were then placed in individual borosilicate reaction vessels and reacted at $77 \pm 1^{\circ} \mathrm{C}$ with four drops of anhydrous phosphoric acid for $8 \mathrm{~min}$ (a total of $12 \mathrm{~min}$ for dolomites, $17 \mathrm{~min}$ for apatite, and 22 min for siderites) in a Finnigan MAT Kiel IV preparation device coupled directly to the inlet of a Finnigan MAT 253 triple collector isotope ratio mass spectrometer. $\mathrm{O}^{17}$ corrected data are corrected for acid fractionation and source mixing by calibration to a best-fit regression line defined by two NBS standards, NBS 18 and NBS 19. Data are reported in \%o notation relative to VPDB. Precision and accuracy of data are monitored through daily analysis of a variety of powdered carbonate standards. At least four standards are reacted and analyzed daily. Measured precision is maintained at better than $0.1 \%$ for both carbon and oxygen isotope compositions.

Martinsburg Fm. turbidites and calcareous siltstone materials were analyzed using X-ray flouresence methods at McGill University, Montreal. These materials were also powdered and 
separated at Macalester to extract detrital zircons; none were recovered. We also attempted to use $\mathrm{U}-\mathrm{Pb}$ laser ablation methods to date the syn-orogenic calcite but this was unsuccessful due to low $\mathrm{U}$ levels and high levels of ${ }^{204} \mathrm{~Pb}$ [38].

\subsection{Calcite Twin Analysis}

The Calcite Strain-Gage Technique (CSGT) of Groshong [39] allows investigation of intraplate stresses as constrained by intracrystalline twinning of rock-forming calcite grains. Although the result is actually a strain tensor, a similar orientation of the stress tensor appears likely in case of coaxial deformation [40,41]. The CSGT has been used to constrain strain tensor directions in veins [21,42,43], limestones [20,26,44-49], marble [50,51], amygdaloidal basalts [52-54], and lamprophryres [55].

Under temperatures of $\mathrm{ca} .200{ }^{\circ} \mathrm{C}$ intracrystalline deformation of calcite results in the formation of e-twins. The formation of calcite e-twins requires a shear stress exceeding ca. $10 \mathrm{MPa}$ [56-59]. Calcite offers three glide systems for e-twinning. From U-stage measurements of width, frequency, and orientation of twins, and the crystallographic orientation of the host crystals, a strain tensor can be calculated using a least-squares technique [39]. In order to remove "noise" from the dataset, a refinement of the calculated strain tensor can be achieved by stripping $20 \%$ twins with highest deviations [60]. This procedure has been used if the number of measured grains were large $(n>20)$. In cases where the data appear to be inhomogeneous, the separation of incompatible twins ("NEV" = negative expected values) from compatible twins ("POS" = positive expected values) of the initial dataset allows separate calculation of two or more least-squares deviatoric strain tensors. Thus, the CSGT can be used to obtain information on superimposed deformations $[39,61]$ and differential stress magnitudes [62,63].

The validity of this stripping procedure was demonstrated in experimental tests where the reliability depends on the overall complexity of deformation and the number of grains with twins [61,64]. The stripping procedure was used in cases of high proportions of NEV and a large number of measured grains. An experimental re-evaluation of the CSGT has shown that measurements of about 50 grains on one thin-section or 25 grains on two mutually-perpendicular thin sections yield the best results $[60,65,66]$. The chance to extract the records of more than two deformations from one dataset is limited when dealing with natural rocks [57]. Individual analyses of veins, matrix, nodules, etc., allows the acquisition of several strain tensors without applying statistical data stripping. The complexity of rotational strains in fault zones has limited the application of this method to the efforts of Gray et al. [67]. Application of the CSGT requires the following assumptions to be valid: (1) low temperatures (dominance of Type I and Type II twins); (2) random c-axis orientations of calcite; (3) homogenous strain; (4) coaxial deformation; (5) volume constancy; (6) low porosity materials, and (7) low strain $(<15 \%)$. If these conditions are not fully met, the underlying dataset of the calculated strain tensor could be biased, modified, or random [66]. Strain tensors were calculated from calcite e-twin datasets using the software package of Evans and Groshong [65].

\subsection{EBSD Methods}

Thin sections were polished using a Buehler Mastermet colloidal silica and Buehler grinderpolisher for $1.5 \mathrm{~h}$ in Beijing. The Lattice Preferred Orientation (LPO) data acquisition was finished on a Hitachi S-3400N-II (Singapore, Singapore) scanning electron microscope mounted with a HKL Channel 5 EBSD detector with the thin section surface inclined at $70^{\circ}$ to the incidental beam. Acceleration voltage was $15 \mathrm{kV}$ and the working distance was $18.4 \mathrm{~mm}$. Optic axis measurements were done on calcite grains using the interactive mode, i.e., the data were collected grain by grain, manually with six to eight kikuchi bands detected. EBSD analysis is finished using the CHANNEL5 analysis software. All the LPO data are plotted on lower hemisphere equal-area Schmidt projections. 


\section{Results}

\subsection{Stable Isotopes}

Various calcite elements were sampled and powdered along the traverse (Figure 1) and these results are presented in Table 1 . The Cambrian Kittatiny limestones are consistent with other early Paleozoic marine carbonates and are consistent across the limb of the syncline (samples 1 and 18). All the various calcite veins and fault fillings are also remarkably uniform $\left(\delta^{13} \mathrm{C}-2.0, \delta^{18} \mathrm{O}-13.3 \mathrm{VPDB}\right)$ across the syncline, are not derived locally from the Kittatiny limestones and have an isotopic signature suggestive of calcite precipitation from warm metamorphic fluids ([68]).

Table 1. Stone Church Syncline Stable Isotope Results.

\begin{tabular}{cccc}
\hline Sample & $\delta$ 13C (VPDB) & $\delta$ 18O (VPDB) & Type \\
\hline 1 & -0.98 & -6.60 & Kittatiny Limestone \\
2 & -1.63 & -11.35 & Martinsburg Fm. vein \\
4 & -1.27 & -13.31 & Martinsburg Fm. vein \\
5 & -1.88 & -13.20 & Martinsburg Fm. vein \\
6 & -2.29 & -13.68 & Martinsburg Fm. vein \\
9 & -1.69 & -13.37 & Martinsburg Fm. vein \\
10 & -1.82 & -13.58 & Martinsburg Fm. vein \\
11 & -1.81 & -13.05 & Martinsburg Fm. vein \\
$12(\mathrm{~A})$ & -1.59 & -13.20 & Martinsburg Fm. vein \\
13 & -1.72 & -13.10 & Martinsburg Fm. vein \\
14 & -1.75 & -13.11 & Martinsburg Fm. vein \\
15 & -2.06 & -13.24 & Martinsburg Fm. vein \\
16 & -2.00 & -13.70 & Martinsburg Fm. vein \\
$17(\mathrm{~B})$ & -2.11 & -12.80 & Martinsburg Fm. vein \\
18 & -0.56 & -6.54 & Kittatiny Limestone \\
\hline
\end{tabular}

\subsection{Martinsburg Fm. Traverse}

We collected oriented calcite phases, bracketed by the underlying Cambrian Kittatiny limestones along a SE-NW traverse through the Stone Church syncline (Figures 1-3; Table 2). The Kittatiny limestones preserve a layer-parallel shortening strain in the direction of tectonic transport with modest strain values and no strain overprint. Synorogenic calcite is found as bedding-parallel veins and gouge zones, cleavage-parallel veins and veins that crosscut bedding, and cleavage-parallel veins in the Martinsburg Fm. turbidites (Figure 2). All of these calcite elements preserve a NW-SE layer-parallel shortening strain ( $n=14$ strain results) with extension normal to Taconic transport, or parallel to the strike of the axial planar cleavage $\left(\mathrm{N} 45^{\circ} \mathrm{E}\right)$. Specifically, for calcite precipitated in a cleavage plane, the shortening axis is normal to the cleavage plane with horizontal extension parallel to the strike (Figure 4). Strain values are modest, there is no strain overprint (low NEVs), and the differential stresses responsible for twinning was -323 bars $(-32 \mathrm{MPa})$.

Across from the powerplant, along the Delaware River and Highway 46 (GPS: $40^{\circ} 54^{\prime} 43.95^{\prime \prime} \mathrm{N}$, $75^{\circ} 4^{\prime} 37.04^{\prime \prime} \mathrm{W}$ ), we observed a series of en echelon, calcite-filled veins (see [43]) that merge with a bedding-parallel calcite-filled fault zone (Figure 3a,b). The en echelon array is filled with sparry calcite which merges into the layers of the fault zone above. Both the en echelon array and fault zone kinematics indicate a normal fault offset $\left(\mathrm{N} 40^{\circ} \mathrm{E}, 40^{\circ} \mathrm{SE}\right.$; Top-down-to-the $\left.\mathrm{SE}\right)$. Six twinning samples record a NW-SE sub-horizontal shortening that is within the plane of the fault (which is parallel to bedding; Figure 4). We combined the six normal fault data sets into one $(n=161)$ and separated positive $(n=135)$ and negative expected values $(n=27)$. The $27 \mathrm{NEV}$ grains represent $14 \%$ of the total and would normally be purged with routine data cleaning [39,60,61]. The contrived NEV result records a subtle twinning strain overprint of sub-vertical shortening (Figure 5). The axial planar cleavage does not crosscut the fault plane or en echelon veins. 

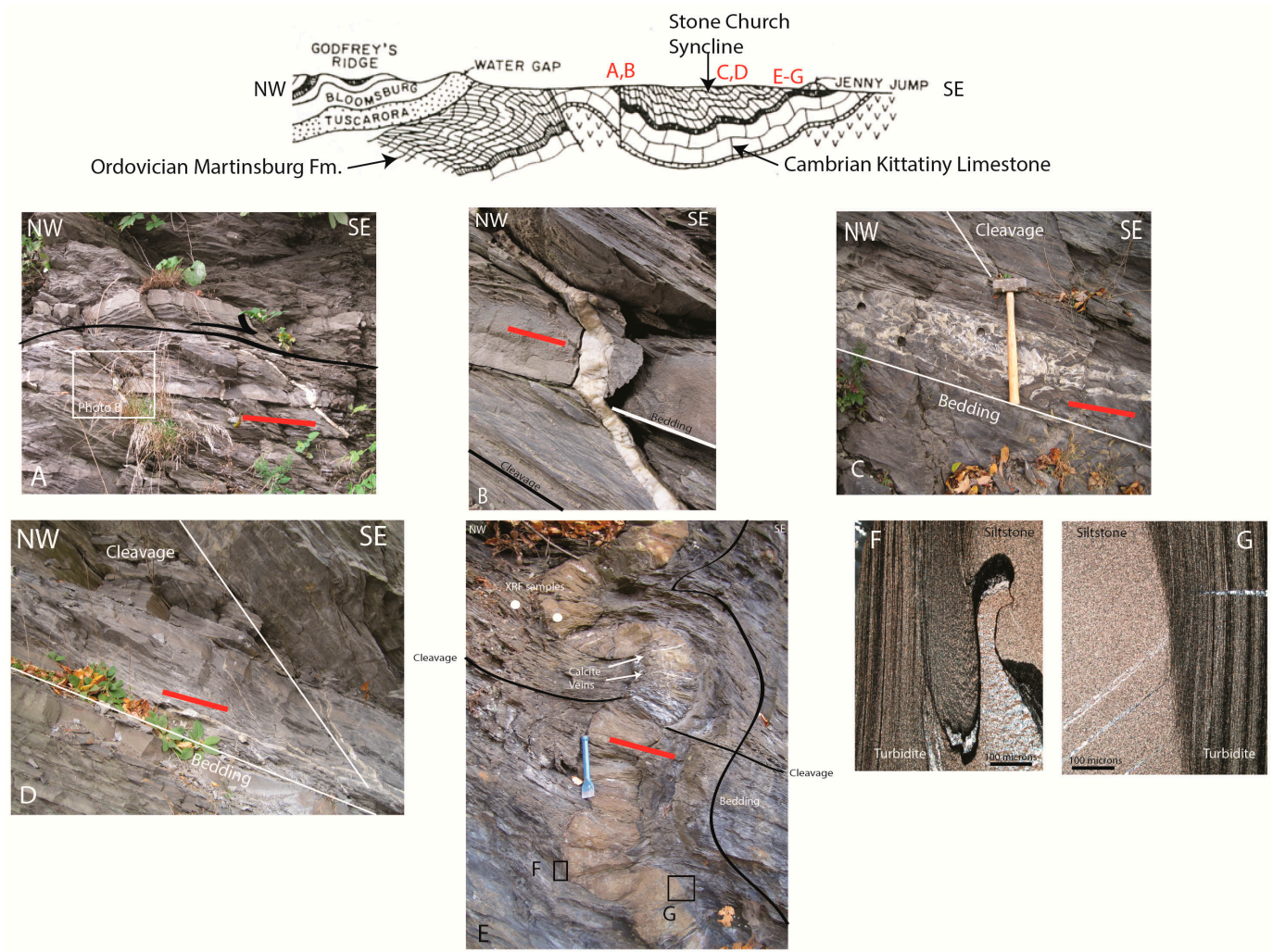

Figure 2. Photo collage of field photos along Maxwell's [4] cross-section (see Figure 1). (A,B) SE-dipping, bedding-parallel normal fault on the northwest limb of the Stone Church syncline; (C,D) bedding and cleavage-parallel calcite fillings; (D-G) the folded, vertical calcareous siltstone layer with horizontal, bedding-normal calcite veins and the locations of the XRF samples (Table 3). The calcareous siltstone layer is part of the Martinsburg turbidite sequence and the cleavage is sheared along the sedimentological interface $(\mathrm{F}, \mathrm{G})$. Dark lines are stylolites and cleavage selvages. Red lines are the orientations of the $\varepsilon 1$ shortening axis for each site.

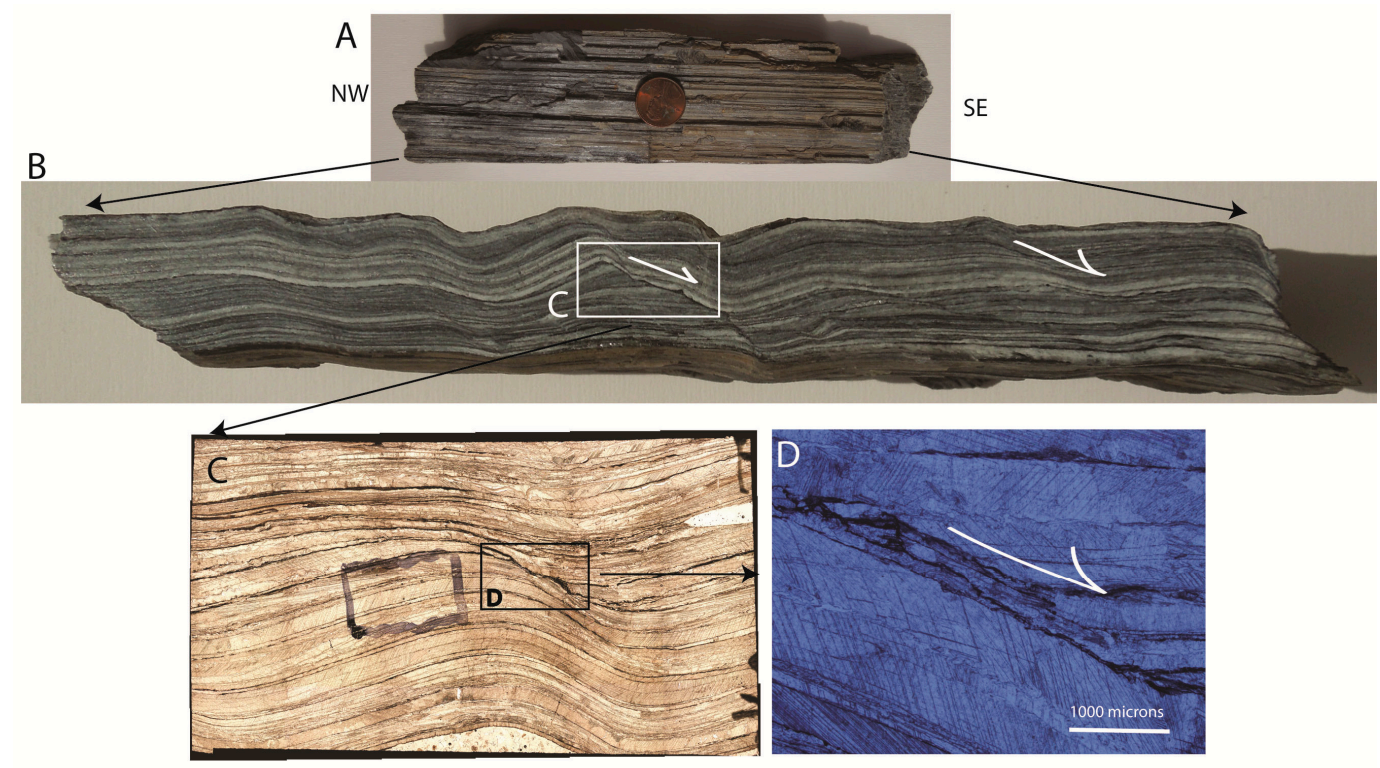

(a)

Figure 3. Cont. 


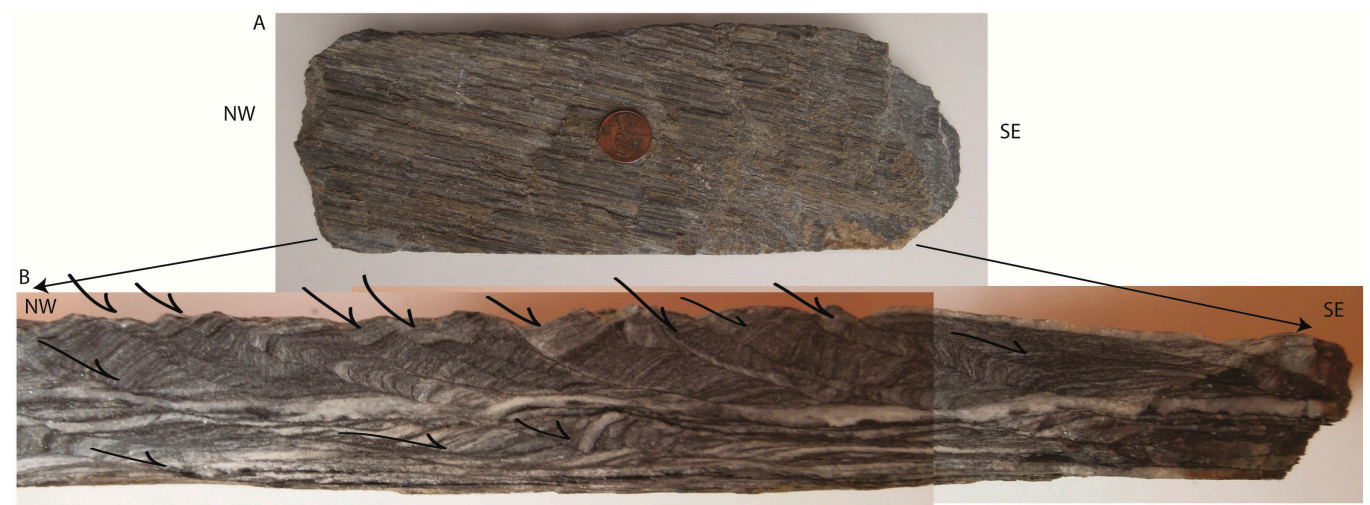

(b)

Figure 3. (a) Normal fault gouge zone with folded fault drag kinematics. Shear zone is grooved (A; top view) and, in cross-section, reveals normal faulted folds (B) shown in thin section (C; full section in plane light) with close-up of fault drag and calcite twins (D; plane light). Coin for scale (3.8 mm diameter); (b) top photo of striated, bedding-parallel normal fault zone with steps down-to-the-SE (A), and a cross-section of the same sample (B) showing normal fault offsets, listric to the local shear zone base.

Shortening Axes for Different Calcite Elements, Martinsburg Fm.
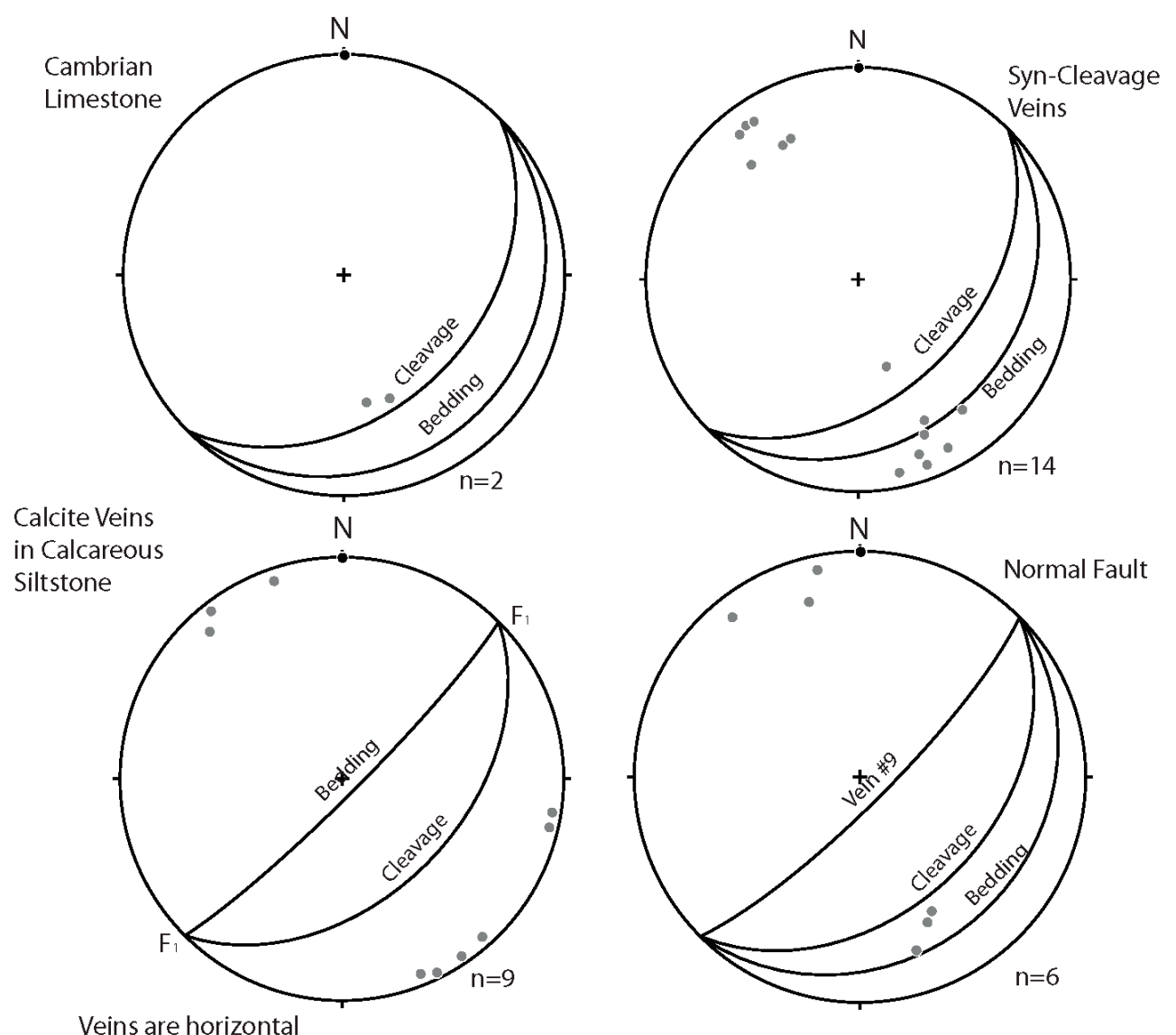

Figure 4. Lower hemisphere projections of calcite twin shortening axes for the various calcite phases, Delaware Water Gap, New Jersey. See Table 2 for details. 
Table 2. Martinsburg Fm. Cleavage-Clastic Dike Calcite Twinning Strain Results.

\begin{tabular}{|c|c|c|c|c|c|c|c|c|c|c|c|c|c|c|}
\hline Sample & $\begin{array}{l}\text { Rock } \\
\text { Unit }\end{array}$ & $\begin{array}{l}\text { Orientation } \\
\text { (Bedding) }\end{array}$ & $\begin{array}{l}\text { Orientation } \\
\text { (Cleavage) }\end{array}$ & $\begin{array}{l}\text { Orientation } \\
\text { (vein) }\end{array}$ & $\begin{array}{c}\text { Grains } \\
(\mathrm{n}=)\end{array}$ & e1 & e2 & e3 & e1 (\%) & $\begin{array}{l}\text { NEV } \\
(\%)\end{array}$ & $-\Delta \sigma$ (bars) & $\begin{array}{l}\text { Fabric } \\
\text { Interp. } \\
\text { (vein) }\end{array}$ & $\begin{array}{c}\text { Fabric } \\
\text { Interp. } \\
\text { (Bedding) }\end{array}$ & Comment \\
\hline \multicolumn{15}{|c|}{ Southeast } \\
\hline 1 & $\begin{array}{l}\text { Kittatiny } \\
\text { LS }\end{array}$ & $\begin{array}{l}\mathrm{N} 45^{\circ} \mathrm{E} \\
38^{\circ} \mathrm{SE}\end{array}$ & None & None & 36 & $\begin{array}{c}171^{\circ} \\
40^{\circ}\end{array}$ & $\begin{array}{c}345^{\circ} \\
62^{\circ}\end{array}$ & $\begin{array}{c}88^{\circ} \\
6^{\circ}\end{array}$ & -3.8 & 19 & 377.76021 & - & LPS & - \\
\hline 2 & $\begin{array}{l}\text { Om, qtz } \\
\text { vein }\end{array}$ & $\begin{array}{l}\mathrm{N} 45^{\circ} \mathrm{E} \\
84^{\circ} \mathrm{SE}\end{array}$ & $\begin{array}{l}\mathrm{N} 45^{\circ} \mathrm{E} \\
42^{\circ} \mathrm{SE}\end{array}$ & - & - & - & - & - & - & - & - & - & - & Fold, no twins \\
\hline $3-1 \mathrm{~A}$ & $\begin{array}{l}\text { Om, } \\
\text { vein }\end{array}$ & $\begin{array}{l}\mathrm{N} 45^{\circ} \mathrm{E} \\
84^{\circ} \mathrm{SE}\end{array}$ & $\begin{array}{l}\mathrm{N} 45^{\circ} \mathrm{E} \\
45^{\circ} \mathrm{SE}\end{array}$ & Horizontal & 30 & $\begin{array}{c}341^{\circ} \\
7^{\circ}\end{array}$ & $\begin{array}{l}163^{\circ} \\
87^{\circ}\end{array}$ & $\begin{array}{l}53^{\circ} \\
12^{\circ}\end{array}$ & -2.9 & 10 & 373.300662 & VPS & LNS & $\begin{array}{l}\text { Fold (Calcareous } \\
\text { siltstone) }\end{array}$ \\
\hline $3-1 B$ & $\begin{array}{l}\text { Om, } \\
\text { vein }\end{array}$ & $\begin{array}{l}\mathrm{N} 45^{\circ} \mathrm{E} \\
84^{\circ} \mathrm{SE}\end{array}$ & $\begin{array}{l}\mathrm{N} 45^{\circ} \mathrm{E} \\
45^{\circ} \mathrm{SE}\end{array}$ & Horizontal & 23 & $\begin{array}{c}101^{\circ} \\
3^{\circ}\end{array}$ & $\begin{array}{c}281^{\circ} \\
87^{\circ}\end{array}$ & $\begin{array}{l}42^{\circ} \\
6^{\circ}\end{array}$ & -1.4 & 21 & 366.464023 & VPS & LNS & $\begin{array}{l}\text { Fold (Calcareous } \\
\text { siltstone) }\end{array}$ \\
\hline $3-2 \mathrm{~A}$ & $\begin{array}{l}\text { Om, } \\
\text { vein }\end{array}$ & $\begin{array}{l}\mathrm{N} 45^{\circ} \mathrm{E} \\
84^{\circ} \mathrm{SE}\end{array}$ & $\begin{array}{l}\mathrm{N} 45^{\circ} \mathrm{E} \\
45^{\circ} \mathrm{SE}\end{array}$ & Horizontal & 8 & $\begin{array}{c}322^{\circ} \\
4^{\circ}\end{array}$ & $\begin{array}{l}143^{\circ} \\
88^{\circ}\end{array}$ & $\begin{array}{c}253^{\circ}, \\
6^{\circ}\end{array}$ & -1.3 & 0 & 370.339565 & VPS & LNS & $\begin{array}{l}\text { Fold (Calcareous } \\
\text { siltstone) }\end{array}$ \\
\hline $3-3 \mathrm{~A}$ & $\begin{array}{l}\text { Om, } \\
\text { vein }\end{array}$ & $\begin{array}{l}\mathrm{N} 45^{\circ} \mathrm{E} \\
84^{\circ} \mathrm{SE}\end{array}$ & $\begin{array}{l}\mathrm{N} 45^{\circ} \mathrm{E} \\
45^{\circ} \mathrm{SE}\end{array}$ & Horizontal & 28 & $\begin{array}{c}155^{\circ} \\
4^{\circ}\end{array}$ & $\begin{array}{c}318^{\circ} \\
88^{\circ}\end{array}$ & $\begin{array}{c}48^{\circ} \\
7^{\circ}\end{array}$ & -1.7 & 10 & 373.7829 & VPS & LNS & $\begin{array}{c}\text { Fold (Calcareous } \\
\text { siltstone) }\end{array}$ \\
\hline $3-4 \mathrm{~A}$ & $\begin{array}{l}\text { Om, } \\
\text { vein }\end{array}$ & $\begin{array}{l}\mathrm{N} 45^{\circ} \mathrm{E} \\
84^{\circ} \mathrm{SE}\end{array}$ & $\begin{array}{l}\mathrm{N} 45^{\circ} \mathrm{E} \\
45^{\circ} \mathrm{SE}\end{array}$ & Horizontal & 42 & $\begin{array}{c}138^{\circ} \\
6^{\circ}\end{array}$ & $\begin{array}{l}317^{\circ} \\
86^{\circ}\end{array}$ & $\begin{array}{l}47^{\circ} \\
16^{\circ}\end{array}$ & -1.8 & 21 & 360.67426 & VPS & LNS & $\begin{array}{l}\text { Fold (Calcareous } \\
\text { siltstone) }\end{array}$ \\
\hline $3-4 \mathrm{~B}$ & $\begin{array}{l}\text { Om, } \\
\text { vein }\end{array}$ & $\begin{array}{l}\mathrm{N} 45^{\circ} \mathrm{E} \\
84^{\circ} \mathrm{SE}\end{array}$ & $\begin{array}{l}\mathrm{N} 45^{\circ} \mathrm{E} \\
45^{\circ} \mathrm{SE}\end{array}$ & Horizontal & 42 & $\begin{array}{c}148^{\circ} \\
5^{\circ}\end{array}$ & $\begin{array}{c}321^{\circ} \\
88^{\circ}\end{array}$ & $\begin{array}{c}54^{\circ} \\
6^{\circ}\end{array}$ & -1.5 & 12 & 373.7829 & VPS & LNS & $\begin{array}{c}\text { Fold (Calcareous } \\
\text { siltstone) }\end{array}$ \\
\hline $3-5 \mathrm{~A}$ & $\begin{array}{l}\text { Om, } \\
\text { vein }\end{array}$ & $\begin{array}{l}\mathrm{N} 45^{\circ} \mathrm{E} \\
84^{\circ} \mathrm{SE}\end{array}$ & $\begin{array}{l}\mathrm{N} 45^{\circ} \mathrm{E} \\
45^{\circ} \mathrm{SE}\end{array}$ & Horizontal & 34 & $\begin{array}{l}318^{\circ} \\
11^{\circ}\end{array}$ & $\begin{array}{c}141^{\circ} \\
87^{\circ}\end{array}$ & $\begin{array}{c}35^{\circ} \\
9^{\circ}\end{array}$ & -1.1 & 29 & 367.25548 & VPS & LNS & $\begin{array}{l}\text { Fold (Calcareous } \\
\text { siltstone) }\end{array}$ \\
\hline $3-5 B$ & $\begin{array}{l}\text { Om, } \\
\text { vein }\end{array}$ & $\begin{array}{l}\mathrm{N} 45^{\circ} \mathrm{E} \\
84^{\circ} \mathrm{SE}\end{array}$ & $\begin{array}{l}\mathrm{N} 45^{\circ} \mathrm{E} \\
45^{\circ} \mathrm{SE}\end{array}$ & Horizontal & 16 & $\begin{array}{c}99^{\circ} \\
8^{\circ}\end{array}$ & $\begin{array}{c}33^{\circ} \\
4^{\circ}\end{array}$ & $\begin{array}{l}272^{\circ} \\
88^{\circ}\end{array}$ & -1.1 & 0 & 402.519949 & VPS & LNS & $\begin{array}{l}\text { Fold (Calcareous } \\
\text { siltstone) }\end{array}$ \\
\hline $\begin{array}{c}\text { 3: } \\
\text { All-PEV }\end{array}$ & $\begin{array}{l}\text { Om, } \\
\text { veins }\end{array}$ & $\begin{array}{l}\mathrm{N} 45^{\circ} \mathrm{E} \\
84^{\circ} \mathrm{SE}\end{array}$ & $\begin{array}{l}\mathrm{N} 45^{\circ} \mathrm{E} \\
45^{\circ} \mathrm{SE}\end{array}$ & Horizontal & 223 & $\begin{array}{c}158^{\circ} \\
4^{\circ}\end{array}$ & $\begin{array}{c}221^{\circ} \\
12^{\circ}\end{array}$ & $\begin{array}{l}342^{\circ}, \\
87^{\circ}\end{array}$ & -7.2 & 0 & 383.247517 & VPS & LNS & $\begin{array}{l}\text { Fold (Calcareous } \\
\text { siltstone) }\end{array}$ \\
\hline $\begin{array}{c}\text { 3: } \\
\text { All-NEV }\end{array}$ & $\begin{array}{l}\text { Om, } \\
\text { veins }\end{array}$ & $\begin{array}{l}\mathrm{N} 45^{\circ} \mathrm{E} \\
84^{\circ} \mathrm{SE}\end{array}$ & $\begin{array}{l}\mathrm{N} 45^{\circ} \mathrm{E} \\
45^{\circ} \mathrm{SE}\end{array}$ & Horizontal & 26 & $\begin{array}{c}36^{\circ} \\
6^{\circ}\end{array}$ & $\begin{array}{c}326^{\circ} \\
2^{\circ}\end{array}$ & $\begin{array}{l}147^{\circ} \\
88^{\circ}\end{array}$ & -3.6 & 100 & 358.639492 & VPS & LPS & $\begin{array}{l}\text { Fold (Calcareous } \\
\text { siltstone) }\end{array}$ \\
\hline 4 & $\begin{array}{l}\text { Om, } \\
\text { vein }\end{array}$ & $\begin{array}{l}\mathrm{N} 45^{\circ} \mathrm{E} \\
20^{\circ} \mathrm{SE}\end{array}$ & $\begin{array}{l}\mathrm{N} 45^{\circ} \mathrm{E} \\
40^{\circ} \mathrm{SE}\end{array}$ & $\begin{array}{l}\mathrm{N} 45^{\circ} \mathrm{E} \\
20^{\circ} \mathrm{SE}\end{array}$ & 29 & $\begin{array}{c}330^{\circ} \\
12^{\circ}\end{array}$ & $\begin{array}{c}225^{\circ} \\
32^{\circ}\end{array}$ & $\begin{array}{c}62^{\circ} \\
3^{\circ}\end{array}$ & -3.7 & 17 & 405.4012551 & VPS & LPS & $\begin{array}{l}\text { Bedding-parallel } \\
\text { normal fault }\end{array}$ \\
\hline 5 & $\begin{array}{l}\text { Om, } \\
\text { vein }\end{array}$ & $\begin{array}{l}\mathrm{N} 45^{\circ} \mathrm{E} \\
20^{\circ} \mathrm{SE}\end{array}$ & $\begin{array}{l}\mathrm{N} 45^{\circ} \mathrm{E} \\
40^{\circ} \mathrm{SE}\end{array}$ & $\begin{array}{l}\mathrm{N} 45^{\circ} \mathrm{E} \\
20^{\circ} \mathrm{SE}\end{array}$ & 33 & $\begin{array}{c}155^{\circ} \\
30^{\circ}\end{array}$ & $\begin{array}{c}326^{\circ} \\
47^{\circ}\end{array}$ & $\begin{array}{c}65^{\circ} \\
4^{\circ}\end{array}$ & -1.4 & 18 & 259.142013 & VPS & LPS & $\begin{array}{l}\text { Bedding-parallel } \\
\text { normal fault }\end{array}$ \\
\hline 6 & $\begin{array}{l}\text { Om, } \\
\text { vein }\end{array}$ & $\begin{array}{l}\mathrm{N} 45^{\circ} \mathrm{E} \\
20^{\circ} \mathrm{SE}\end{array}$ & $\begin{array}{l}\mathrm{N} 45^{\circ} \mathrm{E} \\
40^{\circ} \mathrm{SE}\end{array}$ & $\begin{array}{l}\mathrm{N} 45^{\circ} \mathrm{E} \\
20^{\circ} \mathrm{SE}\end{array}$ & 36 & $\begin{array}{c}162^{\circ} \\
21^{\circ}\end{array}$ & $\begin{array}{c}252^{\circ} \\
12^{\circ}\end{array}$ & $\begin{array}{l}6^{\circ} \\
83^{\circ}\end{array}$ & -1.7 & 5 & 276.1623538 & VPS & LPS & $\begin{array}{l}\text { Bedding-parallel } \\
\text { normal fault }\end{array}$ \\
\hline 7 & $\begin{array}{l}\text { Om, } \\
\text { vein }\end{array}$ & $\begin{array}{l}\mathrm{N} 45^{\circ} \mathrm{E} \\
20^{\circ} \mathrm{SE}\end{array}$ & $\begin{array}{l}\mathrm{N} 45^{\circ} \mathrm{E} \\
40^{\circ} \mathrm{SE}\end{array}$ & $\begin{array}{l}\mathrm{N} 45^{\circ} \mathrm{E} \\
20^{\circ} \mathrm{SE}\end{array}$ & 36 & $\begin{array}{c}349^{\circ} \\
5^{\circ}\end{array}$ & $\begin{array}{c}249^{\circ} \\
83^{\circ}\end{array}$ & $\begin{array}{c}71^{\circ} \\
6^{\circ}\end{array}$ & -2.1 & 1 & 302.2918232 & VPS & LPS & $\begin{array}{l}\text { Bedding-parallel } \\
\text { normal fault }\end{array}$ \\
\hline 8 & $\begin{array}{l}\text { Om, } \\
\text { vein }\end{array}$ & $\begin{array}{l}\mathrm{N} 45^{\circ} \mathrm{E} \\
20^{\circ} \mathrm{SE}\end{array}$ & $\begin{array}{l}\mathrm{N} 45^{\circ} \mathrm{E} \\
40^{\circ} \mathrm{SE}\end{array}$ & $\begin{array}{l}\mathrm{N} 45^{\circ} \mathrm{E} \\
20^{\circ} \mathrm{SE}\end{array}$ & 29 & $\begin{array}{c}342^{\circ} \\
22^{\circ}\end{array}$ & $\begin{array}{c}223^{\circ} \\
61^{\circ}\end{array}$ & $\begin{array}{l}68^{\circ} \\
5^{\circ}\end{array}$ & -1.1 & 10 & 297.7502294 & VPS & LPS & $\begin{array}{l}\text { Bedding-parallel } \\
\text { normal fault }\end{array}$ \\
\hline ALL-PEV & $\begin{array}{l}\text { Om, } \\
\text { vein }\end{array}$ & $\begin{array}{l}\mathrm{N} 45^{\circ} \mathrm{E} \\
20^{\circ} \mathrm{SE}\end{array}$ & $\begin{array}{l}\mathrm{N} 45^{\circ} \mathrm{E} \\
40^{\circ} \mathrm{SE}\end{array}$ & $\begin{array}{l}\mathrm{N} 45^{\circ} \mathrm{E} \\
20^{\circ} \mathrm{SE}\end{array}$ & 163 & $\begin{array}{c}163^{\circ} \\
2^{\circ}\end{array}$ & $\begin{array}{c}253^{\circ} \\
43^{\circ}\end{array}$ & $\begin{array}{l}43^{\circ} \\
5^{\circ}\end{array}$ & -2.7 & 0 & 304.256842 & VPS & LPS & $\begin{array}{l}\text { Bedding-parallel } \\
\text { normal fault }\end{array}$ \\
\hline
\end{tabular}


Table 2. Cont.

\begin{tabular}{|c|c|c|c|c|c|c|c|c|c|c|c|c|c|c|}
\hline Sample & $\begin{array}{l}\text { Rock } \\
\text { Unit }\end{array}$ & $\begin{array}{l}\text { Orientation } \\
\text { (Bedding) }\end{array}$ & $\begin{array}{l}\text { Orientation } \\
\text { (Cleavage) }\end{array}$ & $\begin{array}{l}\text { Orientation } \\
\text { (vein) }\end{array}$ & $\begin{array}{l}\text { Grains } \\
(\mathrm{n}=)\end{array}$ & e1 & e2 & e3 & e1 (\%) & $\begin{array}{l}\text { NEV } \\
(\%)\end{array}$ & $-\Delta \sigma$ (bars) & $\begin{array}{l}\text { Fabric } \\
\text { Interp. } \\
\text { (vein) }\end{array}$ & $\begin{array}{c}\text { Fabric } \\
\text { Interp. } \\
\text { (Bedding) }\end{array}$ & Comment \\
\hline \multicolumn{15}{|c|}{ Southeast } \\
\hline ALL-NEV & $\begin{array}{l}\text { Om, } \\
\text { vein }\end{array}$ & $\begin{array}{l}\mathrm{N} 45^{\circ} \mathrm{E} \\
20^{\circ} \mathrm{SE}\end{array}$ & $\begin{array}{l}\mathrm{N} 45^{\circ} \mathrm{E} \\
40^{\circ} \mathrm{SE}\end{array}$ & $\begin{array}{l}\mathrm{N} 45^{\circ} \mathrm{E} \\
20^{\circ} \mathrm{SE}\end{array}$ & 27 & $\begin{array}{c}168^{\circ} \\
52^{\circ}\end{array}$ & $\begin{array}{l}31^{\circ}, \\
17^{\circ}\end{array}$ & $\begin{array}{c}311^{\circ} \\
18^{\circ}\end{array}$ & -9.9 & 100 & 343.852106 & VNS & LNS & $\begin{array}{c}\text { Bedding-parallel } \\
\text { normal fault }\end{array}$ \\
\hline 9 & $\begin{array}{l}\text { Om, } \\
\text { vein }\end{array}$ & $\begin{array}{l}\mathrm{N} 45^{\circ} \mathrm{E} \\
20^{\circ} \mathrm{SE}\end{array}$ & $\begin{array}{l}\mathrm{N} 45^{\circ} \mathrm{E} \\
40^{\circ} \mathrm{SE}\end{array}$ & $\begin{array}{l}\mathrm{N} 45^{\circ} \mathrm{E} \\
80^{\circ} \mathrm{SE}\end{array}$ & 28 & $\begin{array}{r}152^{\circ} \\
32^{\circ}\end{array}$ & $\begin{array}{c}338^{\circ} \\
28^{\circ}\end{array}$ & $\begin{array}{l}71^{\circ} \\
3^{\circ}\end{array}$ & -1.3 & 0 & 283.807718 & VNS & LPS & S1-normal vein \\
\hline $10 a$ & $\begin{array}{l}\text { Om, } \\
\text { vein }\end{array}$ & $\begin{array}{l}\mathrm{N} 45^{\circ} \mathrm{E} \\
18^{\circ} \mathrm{SE}\end{array}$ & $\begin{array}{l}\mathrm{N} 45^{\circ} \mathrm{E} \\
41^{\circ} \mathrm{SE}\end{array}$ & $\begin{array}{l}\mathrm{N} 45^{\circ} \mathrm{E} \\
18^{\circ} \mathrm{SE}\end{array}$ & 36 & $\begin{array}{c}331^{\circ} \\
28^{\circ}\end{array}$ & $\begin{array}{l}149^{\circ} \\
82^{\circ}\end{array}$ & $\begin{array}{l}73^{\circ} \\
2^{\circ}\end{array}$ & -5.1 & 16 & 279.657792 & VPS & LPS & S1-parallel vein \\
\hline $10 \mathrm{~b}$ & $\begin{array}{l}\text { Om, } \\
\text { vein }\end{array}$ & $\begin{array}{l}\mathrm{N} 45^{\circ} \mathrm{E} \\
18^{\circ} \mathrm{SE}\end{array}$ & $\begin{array}{l}\mathrm{N} 45^{\circ} \mathrm{E} \\
41^{\circ} \mathrm{SE}\end{array}$ & $\begin{array}{l}\mathrm{N} 45^{\circ} \mathrm{E} \\
18^{\circ} \mathrm{SE}\end{array}$ & 36 & $\begin{array}{c}324^{\circ} \\
11^{\circ}\end{array}$ & $\begin{array}{l}128^{\circ} \\
82^{\circ}\end{array}$ & $\begin{array}{c}72^{\circ} \\
6^{\circ}\end{array}$ & -1.9 & 16 & 282.175469 & VPS & LPS & S1-parallel vein \\
\hline 11 & $\begin{array}{l}\text { Om, } \\
\text { vein }\end{array}$ & $\begin{array}{l}\mathrm{N} 45^{\circ} \mathrm{E} \\
18^{\circ} \mathrm{SE}\end{array}$ & $\begin{array}{l}\mathrm{N} 45^{\circ} \mathrm{E} \\
41^{\circ} \mathrm{SE}\end{array}$ & $\begin{array}{l}\mathrm{N} 45^{\circ} \mathrm{E} \\
61^{\circ} \mathrm{SE}\end{array}$ & 36 & $\begin{array}{c}162^{\circ} \\
55^{\circ}\end{array}$ & $\begin{array}{c}26^{\circ} \\
5^{\circ}\end{array}$ & $\begin{array}{c}338^{\circ} \\
12^{\circ}\end{array}$ & -1.8 & 11 & 319.114753 & VPS & LNS & S1-normal vein \\
\hline $12 \mathrm{a}$ & $\begin{array}{l}\text { Om, } \\
\text { vein }\end{array}$ & $\begin{array}{l}\mathrm{N} 45^{\circ} \mathrm{E} \\
15^{\circ} \mathrm{SE}\end{array}$ & $\begin{array}{l}\mathrm{N} 45^{\circ} \mathrm{E} \\
38^{\circ} \mathrm{SE}\end{array}$ & $\begin{array}{l}\mathrm{N} 45^{\circ} \mathrm{E} \\
15^{\circ} \mathrm{SE}\end{array}$ & 34 & $\begin{array}{l}155^{\circ} \\
28^{\circ}\end{array}$ & $\begin{array}{l}252^{\circ} \\
78^{\circ}\end{array}$ & $\begin{array}{l}68^{\circ} \\
3^{\circ}\end{array}$ & -3.3 & 5 & 282.996078 & VPS & LPS & S1-parallel vein \\
\hline $12 \mathrm{~b}$ & $\begin{array}{l}\text { Om, } \\
\text { vein }\end{array}$ & $\begin{array}{l}\mathrm{N} 45^{\circ} \mathrm{E} \\
15^{\circ} \mathrm{SE}\end{array}$ & $\begin{array}{l}\mathrm{N} 45^{\circ} \mathrm{E} \\
38^{\circ} \mathrm{SE}\end{array}$ & $\begin{array}{l}\mathrm{N} 45^{\circ} \mathrm{E} \\
15^{\circ} \mathrm{SE}\end{array}$ & 29 & $152^{\circ}$, & $\begin{array}{l}302^{\circ} \\
82^{\circ}\end{array}$ & $\begin{array}{c}193^{\circ} \\
6^{\circ}\end{array}$ & -1.2 & 3 & 276.162354 & VPS & LPS & S1-parallel vein \\
\hline $13 a$ & $\begin{array}{l}\text { Om, } \\
\text { vein }\end{array}$ & $\begin{array}{l}\mathrm{N} 45^{\circ} \mathrm{E} \\
15^{\circ} \mathrm{SE}\end{array}$ & $\begin{array}{l}\mathrm{N} 45^{\circ} \mathrm{E} \\
38^{\circ} \mathrm{SE}\end{array}$ & $\begin{array}{l}\mathrm{N} 45^{\circ} \mathrm{E} \\
15^{\circ} \mathrm{SE}\end{array}$ & 34 & $\begin{array}{c}161^{\circ} \\
13^{\circ}\end{array}$ & $\begin{array}{c}342^{\circ} \\
52^{\circ}\end{array}$ & $\begin{array}{l}76^{\circ} \\
2^{\circ}\end{array}$ & -1.3 & 3 & 299.73073 & VPS & LPS & S1-parallel vein \\
\hline $13 \mathrm{~b}$ & $\begin{array}{l}\text { Om, } \\
\text { vein }\end{array}$ & $\begin{array}{l}\mathrm{N} 45^{\circ} \mathrm{E} \\
15^{\circ} \mathrm{SE}\end{array}$ & $\begin{array}{l}\mathrm{N} 45^{\circ} \mathrm{E} \\
42^{\circ} \mathrm{SE}\end{array}$ & $\begin{array}{l}\mathrm{N} 45^{\circ} \mathrm{E} \\
42^{\circ} \mathrm{SE}\end{array}$ & 34 & $\begin{array}{c}168^{\circ}, \\
8^{\circ}\end{array}$ & $\begin{array}{l}311^{\circ}, \\
68^{\circ}\end{array}$ & $\begin{array}{l}75^{\circ} \\
4^{\circ}\end{array}$ & -1.3 & 19 & 319.114753 & VPS & LPS & S1-normal vein \\
\hline 14 & $\begin{array}{l}\text { Om, } \\
\text { vein }\end{array}$ & $\begin{array}{l}\mathrm{N} 45^{\circ} \mathrm{E} \\
36^{\circ} \mathrm{SE}\end{array}$ & $\begin{array}{l}\mathrm{N} 45^{\circ} \mathrm{E} \\
58^{\circ} \mathrm{SE}\end{array}$ & $\begin{array}{l}\mathrm{N} 45^{\circ} \mathrm{E} \\
36^{\circ} \mathrm{SE}\end{array}$ & 35 & $\begin{array}{c}323^{\circ} \\
12^{\circ}\end{array}$ & $\begin{array}{c}171^{\circ}, \\
62^{\circ}\end{array}$ & $\begin{array}{c}63^{\circ} \\
8^{\circ}\end{array}$ & -4.8 & 2 & 292.195165 & VPS & LPS & S1-parallel vein \\
\hline 15 & $\begin{array}{l}\text { Om, } \\
\text { vein }\end{array}$ & $\begin{array}{l}\mathrm{N} 45^{\circ} \mathrm{E} \\
36^{\circ} \mathrm{SE}\end{array}$ & $\begin{array}{l}\mathrm{N} 45^{\circ} \mathrm{E} \\
58^{\circ} \mathrm{SE}\end{array}$ & $\begin{array}{l}\mathrm{N} 45^{\circ} \mathrm{E} \\
36^{\circ} \mathrm{SE}\end{array}$ & 36 & $\begin{array}{c}331^{\circ} \\
27^{\circ}\end{array}$ & $\begin{array}{l}133^{\circ} \\
71^{\circ}\end{array}$ & $\begin{array}{l}51^{\circ} \\
11^{\circ}\end{array}$ & -6.1 & 22 & 302.291823 & VPS & LPS & S1-parallel vein \\
\hline $16 a$ & $\begin{array}{l}\text { Om, } \\
\text { vein }\end{array}$ & $\begin{array}{l}\mathrm{N} 45^{\circ} \mathrm{E} \\
36^{\circ} \mathrm{SE}\end{array}$ & $\begin{array}{l}\mathrm{N} 45^{\circ} \mathrm{E} \\
58^{\circ} \mathrm{SE}\end{array}$ & $\begin{array}{l}\mathrm{N} 45^{\circ} \mathrm{E} \\
36^{\circ} \mathrm{SE}\end{array}$ & 37 & $\begin{array}{c}160^{\circ}, \\
8^{\circ}\end{array}$ & $\begin{array}{l}321^{\circ} \\
62^{\circ}\end{array}$ & $\begin{array}{l}70^{\circ} \\
3^{\circ}\end{array}$ & -1.5 & 16 & 305.975108 & VPS & LPS & S1-parallel vein \\
\hline $16 \mathrm{~b}$ & $\begin{array}{l}\text { Om, } \\
\text { vein }\end{array}$ & $\begin{array}{l}\mathrm{N} 45^{\circ} \mathrm{E} \\
36^{\circ} \mathrm{SE}\end{array}$ & $\begin{array}{l}\mathrm{N} 45^{\circ} \mathrm{E} \\
58^{\circ} \mathrm{SE}\end{array}$ & $\begin{array}{l}\mathrm{N} 45^{\circ} \mathrm{E} \\
36^{\circ} \mathrm{SE}\end{array}$ & 35 & $\begin{array}{c}326^{\circ} \\
11^{\circ}\end{array}$ & $\begin{array}{c}168^{\circ} \\
82^{\circ}\end{array}$ & $\begin{array}{l}61^{\circ} \\
5^{\circ}\end{array}$ & -4.9 & 0 & 281.345691 & VPS & LPS & S1-parallel vein \\
\hline $17 \mathrm{a}$ & $\begin{array}{l}\text { Om, } \\
\text { vein }\end{array}$ & $\begin{array}{l}\mathrm{N} 45^{\circ} \mathrm{E} \\
21^{\circ} \mathrm{SE}\end{array}$ & $\begin{array}{l}\mathrm{N} 45^{\circ} \mathrm{E} \\
65^{\circ} \mathrm{SE}\end{array}$ & $\begin{array}{l}\mathrm{N} 45^{\circ} \mathrm{E} \\
21^{\circ} \mathrm{SE}\end{array}$ & 28 & $\begin{array}{l}318^{\circ} \\
28^{\circ}\end{array}$ & $\begin{array}{c}147^{\circ} \\
56^{\circ}\end{array}$ & $\begin{array}{l}69^{\circ} \\
8^{\circ}\end{array}$ & -5.3 & 17 & 277.930636 & VPS & LPS & S1-parallel vein \\
\hline $17 \mathrm{~b}$ & $\begin{array}{l}\text { Om, } \\
\text { vein }\end{array}$ & $\begin{array}{l}\mathrm{N} 45^{\circ} \mathrm{E} \\
21^{\circ} \mathrm{SE}\end{array}$ & $\begin{array}{l}\mathrm{N} 45^{\circ} \mathrm{E} \\
65^{\circ} \mathrm{SE}\end{array}$ & $\begin{array}{l}\mathrm{N} 45^{\circ} \mathrm{E} \\
21^{\circ} \mathrm{SE}\end{array}$ & 36 & $\begin{array}{c}141^{\circ} \\
22^{\circ}\end{array}$ & $\begin{array}{l}251^{\circ} \\
72^{\circ}\end{array}$ & $\begin{array}{l}72^{\circ} \\
5^{\circ}\end{array}$ & -4.9 & 8 & 269.619124 & VPS & LPS & S1-parallel vein \\
\hline $17 \mathrm{c}$ & $\begin{array}{l}\text { Om, } \\
\text { vein }\end{array}$ & $\begin{array}{l}\mathrm{N} 45^{\circ} \mathrm{E} \\
21^{\circ} \mathrm{SE}\end{array}$ & $\begin{array}{l}\mathrm{N} 45^{\circ} \mathrm{E} \\
65^{\circ} \mathrm{SE}\end{array}$ & $\begin{array}{l}\mathrm{N} 45^{\circ} \mathrm{E} \\
21^{\circ} \mathrm{SE}\end{array}$ & 28 & $\begin{array}{c}157^{\circ} \\
22^{\circ}\end{array}$ & $\begin{array}{c}328^{\circ} \\
51^{\circ}\end{array}$ & $\begin{array}{c}66^{\circ} \\
8^{\circ}\end{array}$ & -3.3 & 13 & 293.62337 & VPS & LPS & S1-parallel vein \\
\hline 18 & $\begin{array}{c}\text { Kittatiny } \\
\text { LS }\end{array}$ & $\begin{array}{l}\mathrm{N} 45^{\circ} \mathrm{E} \\
14^{\circ} \mathrm{SE}\end{array}$ & None & None & 31 & $\begin{array}{c}160^{\circ}, \\
42^{\circ}\end{array}$ & $\begin{array}{c}352^{\circ} \\
8^{\circ}\end{array}$ & $\begin{array}{r}282^{\circ} \\
28^{\circ}\end{array}$ & -1.8 & 25 & 339.5959185 & - & LPS & - \\
\hline \multicolumn{15}{|c|}{ Northwest } \\
\hline- & - & - & - & - & $\mathrm{n}=1341$ & - & - & - & $\begin{array}{c}\text { Avg.: } \\
-2.9 \%\end{array}$ & - & $\begin{array}{c}\text { Avg: } \\
-323 \text { bars }\end{array}$ &.- & - & - \\
\hline
\end{tabular}

Om: Ordovician Martinsburg Fm. Fabric Interpretation: LPS (layer-parallel shortening); VPS (vein-parallel shortening); VNS (vein-normal shortening). 

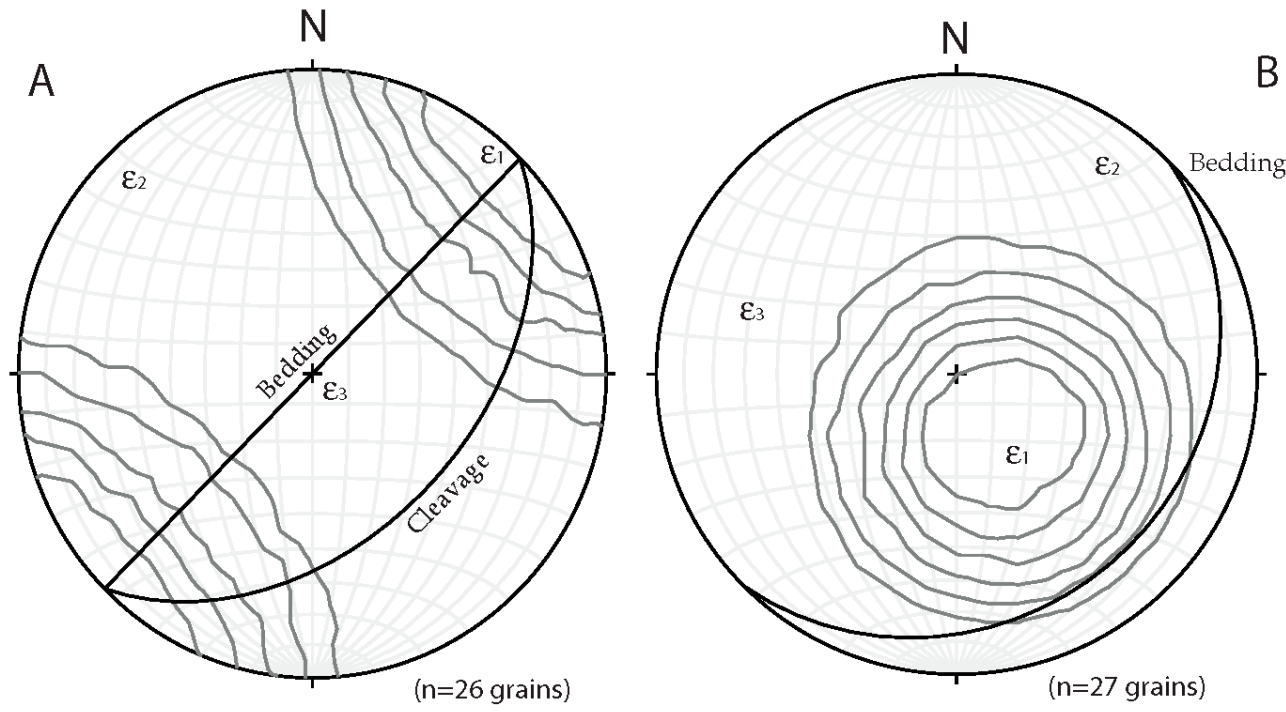

Figure 5. Lower hemisphere stereoplots of "contrived" strain overprint data (NEVs) for (A) the calcite veins in the calcareous siltstone and (B) the bedding-parallel normal fault. Strain axes (E1 = shortening, E2 = intermediate axis, E3 = extension) are plotted with Turner [40] compression axes contoured. See Table 2 .

\subsection{Calcareous Siltstone Layers}

We sampled the Martinsburg Fm. turbidites and an interbedded calcareous siltstone across a concordant depositional contact (Figure 2). Both major (e.g., $\mathrm{SiO}_{2}, \mathrm{Al}_{2} \mathrm{O}_{3}, \mathrm{CaO}$, LOI) and trace element (e.g., $\mathrm{Rb}, \mathrm{Sr}$ ) counts are remarkably different (Table 3). Heavy mineral separations, with the intent of using detrital zircons for provenance and depositional age data (see [69]) resulted in the recovery of pyrite and no zircons from either sample.

One large oriented hand sample of a calcareous siltstone was collected north of the classic roadcut (Figure 2; see Maxwell, [4], Figures 9 and 10). This sample was cut into five vertical slabs from which two oriented thin sections per slab were recovered for a total of 10 thin sections. Bedding is sub-vertical and the horizontal veins are bed-normal. Petrographic observations reveal a sheared boundary between the turbidites and siltstone, local vein and stylolite development, and poor cleavage development in the siltstone (Figure $2 \mathrm{e}-\mathrm{g}$ ). Calcite twin analysis revealed a uniform sub-horizontal shortening strain that is normal to the local bedding (layer-normal shortening) and fold axis, yet within the locally-horizontal cleavage (Figure 2, Table 2). For the combined data set, the strain overprint was negligible (low NEVs; $14 \%$ ) and removed with routine data cleaning, but we did combine 26 NEV grains for a separate strain analysis. This contrived strain overprint records a sub-horizontal shortening strain approximately parallel to the Stone Church syncline fold axis and Taconic margin (SW-NE; Figure 5).

EBSD fabric results for the siltstone layer show a robust Lattice Preferred Orientation (LPO), even though the calcite grains (10-50 microns) are widely spaced ( 200 microns) in the matrix between stylolite seams implying that the deformation was homogeneous on the scale of the thin section. The calcite $(<001>)$ axes are sub-horizontal and parallel to the Taconic shortening direction (NW-SE), similar to the a-axes $(<11-20>)$, both of which are similar to the fabrics of calcite polycrystals (both experimentally and naturally deformed) at low temperature $\left(\sim 100^{\circ} \mathrm{C}\right)$ reported by Wenk et al. [56,70] (Figure 6). The inferred horizontal shortening is also perpendicular to bedding, which is sub-vertical. 
Table 3. Whole-rock geochemistry (XRF).

\begin{tabular}{|c|c|c|c|}
\hline \multirow{2}{*}{-} & \multicolumn{3}{|c|}{ Samples } \\
\hline & Martinsburg Fm. (Calc. siltstone) & Martinsburg Fm. & Detection Limits (ppm) \\
\hline \multicolumn{4}{|c|}{ Major Elements } \\
\hline $\mathrm{SiO}_{2}$ & 18.32 & 56.64 & 60 \\
\hline $\mathrm{TiO}_{2}$ & 0.2989 & 0.8694 & 25 \\
\hline $\mathrm{Al}_{2} \mathrm{O}_{3}$ & 6.2 & 18.16 & 120 \\
\hline $\mathrm{Fe}_{2} \mathrm{O}_{3}$ & 9.359 & 7.2402 & 25 \\
\hline $\mathrm{MnO}$ & 0.3959 & 0.0494 & 25 \\
\hline $\mathrm{MgO}$ & 11.3 & 3.26 & 95 \\
\hline $\mathrm{CaO}$ & 20.61 & 2.14 & 15 \\
\hline $\mathrm{Na}_{2} \mathrm{O}$ & 0.2208 & 0.9132 & 35 \\
\hline $\mathrm{K}_{2} \mathrm{O}$ & 1.29 & 4.45 & 25 \\
\hline $\mathrm{P}_{2} \mathrm{O}_{5}$ & 0.376 & 0.158 & 35 \\
\hline $\mathrm{BaO}$ & 1113.2 & 750.3 & 12 \\
\hline $\mathrm{Ce}$ & 42 & 104 & 15 \\
\hline Co & $<\mathrm{d} / 1$ & 15 & 10 \\
\hline $\mathrm{Cr}_{2} \mathrm{O}_{3}$ & 51.3 & 138.3 & 10 \\
\hline $\mathrm{Cu}$ & 31 & 50 & 2 \\
\hline $\mathrm{Ni}$ & 12 & 45 & 3 \\
\hline $\mathrm{Sc}$ & 15 & 13 & 7 \\
\hline V & 71.4 & 129.9 & 7 \\
\hline $\mathrm{Zn}$ & 43 & 81 & 2 \\
\hline LOI & 31.34 & 5.84 & 100 \\
\hline Total & 99.85 & 99.85 & - \\
\hline \multicolumn{4}{|c|}{ Trace Elements } \\
\hline $\mathrm{Ga}$ & 7.1 & 23.9 & 1 \\
\hline $\mathrm{Nb}$ & 5.8 & 16.6 & 0.3 \\
\hline $\mathrm{Pb}$ & 2.8 & 19 & 1 \\
\hline $\mathrm{Rb}$ & 53.3 & 171.8 & 1 \\
\hline $\mathrm{Sr}$ & 723.6 & 100 & 1 \\
\hline Th & 3.7 & 8.5 & 1 \\
\hline $\mathrm{U}$ & 1.9 & 3.6 & 1 \\
\hline $\mathrm{Y}$ & 24.6 & 19.9 & 1 \\
\hline $\mathrm{Zr}$ & 60.7 & 161 & 1 \\
\hline
\end{tabular}

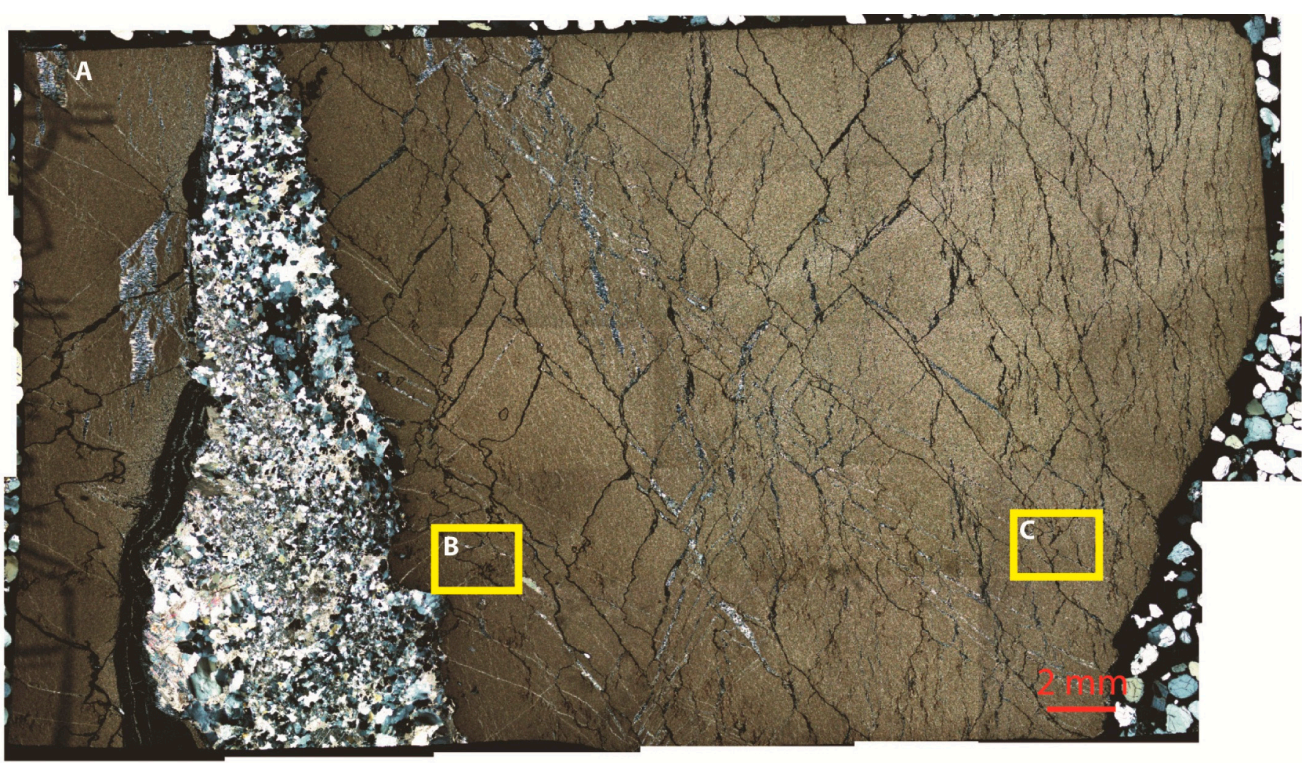

Figure 6. Cont. 


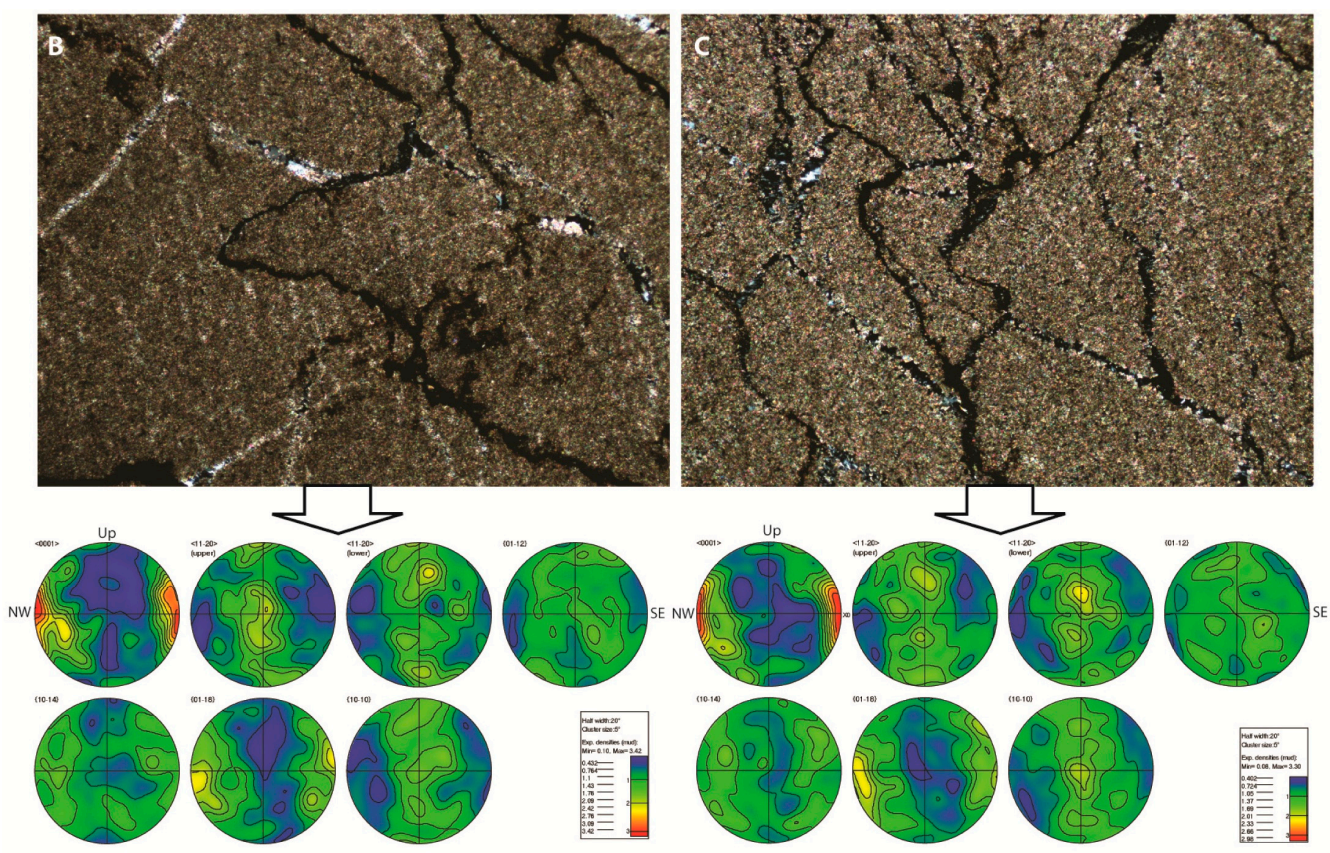

Figure 6. Photomicrograph of calcareous siltstone in the Martinsburg Fm. (A: polarized light) with yellow rectangles indicating areas of EBSD analysis $(\mathbf{B}, \mathbf{C})$ and lower hemisphere pole figure calcite results below ( $\mathrm{n}=202$ for each area, A,B).

\section{Discussion}

Studies of deformation in the Martinsburg Fm., including along the Delaware Water Gap, is quite comprehensive, although few of the published results include oriented strain data. Fold shortening, by bed length curvature calculations, accounts for $\sim 40 \%$ of the shortening in the Delaware Water Gap section [4] which is comparable to the $\sim 50 \%$ regional shortening of a thin-skinned thrust belt. Volume loss by pressure solution during orogenic cleavage formation is well-documented in Appalachian Paleozoic rocks, ranging from $40 \%-75 \%[5-7,19]$ a value similar to the $10 \%-25 \%$ AMS proxy value reported by Pares and van der Pluijm [37]. Recrystallization, observed as pressure shadows parallel to cleavage planes and new chlorite growth, is a significant portion of the Martinsburg deformation [35] and involved a Permian remagnetization [36]. Beutner [7], using 80 measurements on chlorite, report no grain rotation, a result not in agreement with our EBSD measurements in the calcareous siltstones of the Martinsburg Fm. (see below).

Our contribution to understanding the partitioning of strain is the analysis of the five phases of secondary calcite in the Martinsburg Fm. The various calcite phases reported are of a uniform stable isotopic signature and presumably from the same hot, synorogenic Taconic source (Table 1). Calcite twinning, then, represents a minor portion of the total Taconic strain $(-3 \%$ shortening) partitioning in these rocks, with a uniform SE-NW sub-horizontal shortening strain preserved by the secondary calcite across the Stone Church syncline. Strain magnitudes and differential stresses ( $-32 \mathrm{MPa}$ ) are typical of the internal portions of a thrust belt [71] and there is no twinning strain overprint (low NEVs). If the five calcite phases in the Martinsburg Fm. are the result of fluid migration during the Taconic orogeny then we might expect to see a twinning strain related to Acadian terrane accretion [72] and/or the collisional Alleghenian orogeny in the Permian [73,74]. Twinning strain overprints require a significantly different stress field orientation as calcite strain hardens [64] so the Acadian or Alleghenian stress fields were likely parallel (co-axial) to the Taconic stress-strain field. On a regional scale our Piedmont twinning shortening strain $\left(\mathrm{N} 40^{\circ} \mathrm{W}\right)$ is parallel (co-axial) to other twinning and finite strains across the Valley and Ridge and foreland provinces (Figure 7, [8,10,24,25,44,45,75]). The parallelism of layer-parallel shortening fabrics from the Piedmont, across the Valley and Ridge province, and far 
into the foreland ( $2000 \mathrm{~km}$; $[26,27])$ suggests that the pre-Appalachian sedimentary prism (foreland + restored orogenic belt) approaches $\sim 3000 \mathrm{~km}$ and there is little rotation (no vertical axis rotations) between the LPS fabrics in the allochthonous rocks versus the same pre-thrusting LPS fabric in the autochthonous foreland $[8,10,21]$. This result suggests that the Pennsylvania orocline (salient) formed in response to a pre-orogenic margin shape that controlled the structural evolution of the Appalachians. Kinematic data across and around the Valley and Ridge province in the Pennsylvania salient supports a rotational fault-fold genesis in two stages $[9,76]$ a model not supported by the calcite strain data herein. The intense cleavage spacing in the Martinsburg Fm. precludes acquisition of a reliable Ordovician paleomagnetic paleopole and Housen et al. [36] report a complex, four-component paleopole in the Martinsburg Fm. near Lehigh Gap, PA. The mid-late Paleozoic sediments of the Appalachian Valley and Ridge and foreland provinces preserve a late Paleozoic chemical remagnetization overprint making the role of pre-thrusting salient shapes difficult to interpret relative to thrust sheet rotations and migrations [77-85]. These complex data then preclude addressing the role of thrust sheet rotations in the Piedmont with younger magnetic signatures preserved in younger Paleozoic sediments inboard of the hinterland.

Finite strains in the Martinsburg Fm. are reported with aspect ratios up to 5:1 ([4], quartz) with the long axis of the ellipsoidal shape parallel to the cleavage plane dip direction, with shortening normal to the cleavage plane. Syn-cleavage calcite twins record a NW-SE shortening strain, normal to the cleavage strike and parallel to the inferred Taconic thrust transport direction. Extension axes are sub-horizontal and strike-parallel, a result identical to that reported for syn-cleavage twinning strains in the Permian Gondwanide orogeny in the Cape belt, S. Africa [17]. In both cases, the shaly rocks are folding as part of a thrust belt while the rocks are losing volume to pressure solution while syn-orogenic calcite is precipitating along bedding and cleavage planes. Most of the synorogenic calcite is sparry and twins immediately after precipitation, leaving a mechanical paradox: how does a cleavage plane open (dilate) while filling with calcite that shortens normal to the dilation direction? This strain-cleavage relationship is observed everywhere in the Stone Church syncline except on the southeast fold limb in the vertical calcareous siltstone layer within the turbidite sequence (see below).

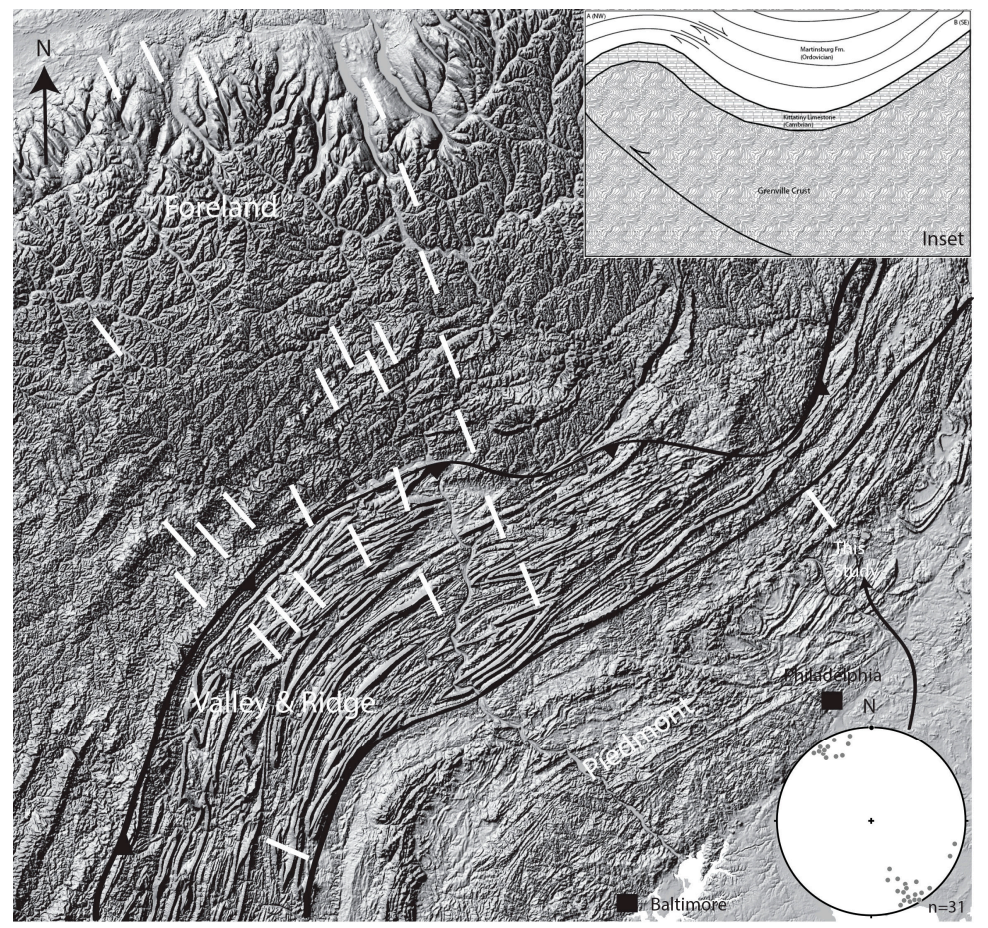

Figure 7. Strain data for the Piedmont, Valley and Ridge, and Foreland provinces across the central Appalachian Mountains and the Pennsylvania Salient orocline. Sources are $[8,10,24,25,44,45,85]$ and this study. Stereonet is a summary plot of calcite twinning shortening axes. Inset is a schematic of a flexural-slip interpretation of Figures $2 \mathrm{a}, \mathrm{b}$ and $3 \mathrm{a}, \mathrm{b}$ and the Solor Church folds. 
Calcareous siltstone layers $(<0.5 \mathrm{~m})$ are infrequent within the Martinsburg Fm. and are part of the Ordovician turbidite sequence. These layers are geochemically different than the enclosing turbidites (Table 3) and there is literature about non-tabular clastic enclaves and dikes in the Martinsburg Fm. [4-6,86]. Isotopic values for calcite veins in this siltstone layer are similar to the other syn-orogenic calcite fluids (Table 1) and the twinning strains correlate well within the multi-layer sample and with twinning strain results across the syncline (Figure 4). Shear between the Martinsburg turbidite and calcareous siltstone demonstrates the rheological difference between these materials relative to the contemporaneous cleavage and the presence of minor folds in the Stone Church syncline. Internally, the fine-grained calcite in the silty layer has a preferred orientation parallel to the inferred Taconic shortening orientation (Figures 2 and 4) but normal to bedding. The calcareous siltstone layer LPO strain proxy and calcite vein twinning shortening strain are sub-horizontal and oriented SE-NW, and both preserve a layer-normal shortening suggesting the strain was imposed in these rocks after the bedding was folded into a sub-vertical orientation as part of the Stone Church syncline. The calcite shortening strain here is parallel to the cleavage dip direction and the extension axis is vertical. The contrived NEV calcite twinning overprint $(n=26)$ preserves a sub-horizontal shortening strain that is parallel to the Stone Church syncline axis, which is roughly parallel to the strike of normal faults on the margin of the Triassic Newark basin (Figure 5). Is this strain overprint related to Acadian $[14,15,87]$ or Triassic [88] strike-slip motion within the Piedmont?

The youngest structural features observed along the Martinsburg Fm. traverse include a few bedding-parallel shear zones $\left(\mathrm{N} 40^{\circ} \mathrm{E}, 25^{\circ} \mathrm{SE}\right)$ with layered calcite gouge zones that are not crosscut by the axial-planar cleavage. These SE-dipping fault zones are more common on the northwest end of the traverse where the northwest limb of the Stone Church syncline rolls into the adjacent anticline (Figure 2). Sparry, en echelon fillings are materially continuous into the layered fault zone and both preserve a top-down-to-the-southeast kinematic sense, indicating that this normal faulting (N40 $\mathrm{E}$, $45^{\circ} \mathrm{SE}$ ) post-dated cleavage formation. Beutner et al. [6] describe calcite boudins that look like normal fault steps (their Figure 6) and folded calcite veins (their Figure 3) that look like the normal fault offsets we describe in Figure 2e-g. This fault zone calcite is chemically similar to the other phases of syn-orogenic calcite (Table 1) and records a NW-SE horizontal shortening strain (Table 2, Figure 4). For a section of NW-vergent folds in the Martinsburg to have SE-dipping normal fault offsets (Figure 3), with a horizontal shortening strain, seems out of place in a Taconic thrust sheet. One explanation could be that the bedding-parallel slip surfaces are zones of flexural-slip within the Martinsburg Fm. and are slip surfaces at the inflection point along the common limb of the Stone Church syncline and the anticline to the northwest (Figure 2). So, for each SE-dipping slip surface, material motion would be down-to-the-SE into the hinge of the syncline (out of the anticline), and the lower surface would move material up-to-the-NW into the (poorly exposed) anticline. If the field observation is taken at face value, as an individual exposure, the stress-strain field of the syn-faulting calcite is not in agreement with the Anderson [89] expectation for principal stress planes and normal faults. We also have evidence of a (contrived) twinning strain overprint in this normal fault zone (Figure 5), a shortening strain that is sub-vertical. Vertical shortening strains are reported in tectonic settings with nappe burial $[17,90]$ but the normal fault zone that crosscuts the Martinsburg Fm. cleavage is the youngest calcite phase, making a vertical shortening strain related to Taconic nappe (e.g., Reading Prong) burial unlikely. A sub-vertical twinning strain related to Triassic extension is a possibility as the youngest strain event, but this result is not in agreement with the NE-trending $\left(5^{\circ}-60^{\circ}\right.$ plunges) shortening result reported by Lomando and Engelder [88] for calcite cements in the Newark Basin New Brunswick sandstones. Based on one detailed outcrop description from the hinge of a Taconic fold, the twinning strains (and contrived strain overprint) suggest a complex stress-strain field related to flexural-slip dynamics again (see inset, Figure 7), similar to the results reported in the Cape belt in Southern Africa [17] who reported a layer-normal twinning strain overprint where these secondary shortening axes rotated through the fold axis during folding. 


\section{Conclusions}

As the Martinsburg Fm. folded, translated, and cleaved (fold axis: N40 ${ }^{\circ}$ ) from SE to NW during the Taconic orogeny five phases of syn-orogenic calcite, of a uniform isotopic signature, precipitated throughout the Stone Church syncline and was mechanically twinned. The calcite strains record a uniform sub-horizontal shortening parallel to the Taconic thrust translation direction (SE-NW), a shortening fabric that is generally parallel to Martinsburg Fm. bedding and at a steep angle to the axial planar cleavage, but normal to the cleavage strike. Extension axes were horizontal during cleavage formation. This result is identical to that reported by Craddock et al. [17] for syn-cleavage Gondwanide deformation calcite twinning strains in the frontal Cape belt. Twinning strains in cleavage planes within the vertical calcareous siltstones also record a SE-NW sub-horizontal shortening strain that is bedding-normal and cleavage-parallel. This relationship indicates a consistent stress-strain field during development of the axial-planar cleavage but rotation of the (minor) fold during formation of the syncline. Some SE-dipping bedding planes contain down-to-the-SE normal fault offsets that are post-cleavage and are likely related to flexural-slip dynamics at the inflection point between migrating Taconic anticlines and synclines and not the Triassic evolution of the Newark-Gettysburg basin.

Acknowledgments: John P. Craddock first visited the Appalachian's on a University of Michigan field trip with Dave Wiltschko in 1981 where Craddock first began to learn about microstructures, calcite twinning and thrust belts. This project also benefited from conversations, over the years, with Cam Craddock about the relations of cleavage formation and clastic dike intrusion, particularly in Michigan's upper peninsula. Jakob Wartman and Maria Princen were funded by Beltmann grants at Macalester. Mary Beth Gray and John Suppe helped us with finding specific contacts in and around the Delaware Water Gap. Field assistance of Annie Craddock and Kristina Holm and photographic creation of Figure $4 \mathrm{a}$, b by Elizabeth Longhurst is greatly appreciated. Reviews by Rick Groshong and three anonymous reviewers greatly improved the clarity of the paper.

Author Contributions: John P. Craddock conceived the project and undertook the fieldwork. John P. Craddock, Maria Princen and Jakob Wartman measured the twinning strains and the EBSD work was perfomed by Haoran Xia and Junlai Liu. John P. Craddock wrote and revised the manuscript.

Conflicts of Interest: The authors declare no conflict of interest.

\section{References}

1. Chapple, W.M. Mechanics of thin-skinned fold-and-thrust belts. Geol. Soc. Am. Bull. 1978, 89, 1189-1198. [CrossRef]

2. Marshak, S. Salients, recesses, arcs, oroclines, and syntaxes-A review of ideas concerning the formation of map-view curves in fold-thrust belts. In Thrust Tectonics and Hydrocarbon Systems: American Association of Petroleum Geologists Memoir 82; McClay, K.R., Ed.; American Association of Petroleum Geologists: Tulsa, OK, USA, 2004; pp. 1-26.

3. Sussman, A.J.; Weil, A.B. Orogenic Curvature: Integrating Paleomagnetic and Structural Analyses; Geological Society of America Special Paper 383; Geological Society of America: Boulder, CO, USA, 2004.

4. Maxwell, J.C. Origin of slaty and fracture cleavage in the Delaware Water Gap are, New Jersey and Pennsylvania. In Petrologic Studies: A Volume in Honor of A.F. Buddington; Engel, A.E.J., James, H.L., Leonard, B.F., Eds.; Geological Society of America: Boulder, CO, Canada, 1962; pp. 281-311.

5. Groshong, R.H., Jr. Strain and pressure solution in the Martinsburg slate, Delaware Water Gap, New Jersey. Am. J. Sci. 1976, 276, 1131-1146. [CrossRef]

6. Beutner, E.C.; Jancin, M.D.; Simon, R.W. Dewatering origin of cleavage in light of deformed calcite veins and clastic dikes in Martinsburg slate, Delaware Water Gap, New Jersey. Geology 1977, 5, 118-122. [CrossRef]

7. Beutner, E.C. Slate cleavage and related strain in Martinsburg slate, Delaware Water Gap, New Jersey. Am. J. Sci. 1978, 278, 1-23. [CrossRef]

8. Ong, P.F.; van der Pluijm, B.A.; van der Voo, R. Early rotation in the Pennsylvania salient (US Appalachians): Evidence from calcite-twinning analysis of Paleozoic carbonates. Geol. Soc. Am. Bull. 2007, 119, 796-804. [CrossRef]

9. Wise, D.U. Pennsylvania salient of the Appalachian's: A two-azimuth transport model based on new compilations of Piedmont data. Geology 2004, 32, 777-780. [CrossRef] 
10. Sak, P.B.; McQuarrie, N.; Oliver, B.P.; Lavdovsky, N.; Jackson, M.S. Unraveling the central Appalachian fold-thrust belt, Pennsylvania of sequentially restored balanced cross sections for a blind fold-thrust belt. Geosphere 2012, 8, 685-702. [CrossRef]

11. Rodgers, J. The Tectonics of the Appalachians; Wiley-Interscience: New York, NY, USA, 1970; p. 271.

12. Thomas, W.A. Evolution of Appalachian salients and recesses from reentrants and promontories in the continental margin. Am. J. Sci. 1977, 277, 1233-1278. [CrossRef]

13. Hatcher, R.D., Jr. Tectonic synthesis of the U.S. Appalachians. In The Appalachian-Ouachita Orogen in the United States; Hatcher, R.D., Jr., Thomas, W.A., Viele, G.W., Eds.; Geological Society of America: Boulder, CO, USA, 1989; pp. 275-288.

14. Faill, R.T. A geologic history of the north-central Appalachians; Part 1, Orogenesis from the Mesoproterozoic through the Taconic Orogeny. Am. J. Sci. 1997, 297, 551-619. [CrossRef]

15. Faill, R.T. A geologic history of the north-central Appalachians; Part 2, The Appalachian Basin from the Silurian through Carboniferous. Am. J. Sci. 1997, 297, 729-761. [CrossRef]

16. Hatcher, R.D., Jr. The Appalachian orogeny: A brief summary. Geol. Soc. Am. Mem. 2010, 206, 1-19.

17. Craddock, J.P.; McKiernan, A.; de Wit, M. Calcite twin analysis in synorogenic calcite, Cape Fold Belt: Implications for fold rotation and cleavage formation. J. Struct. Geol. 2007, 27, 1100-1113. [CrossRef]

18. Cloos, E. Oolite deformation in the South Mountain fold, Maryland. Geol. Soc. Am. Bull. 1947, 58, 843-918. [CrossRef]

19. Wright, T.O.; Platt, L.B. Pressure solution and cleavage in the Martinsburg shale. Am. J. Sci. 1982, 282, 122-135. [CrossRef]

20. Wiltschko, D.V.; Medwedeff, D.A.; Millson, H.E. Distribution and mechanisms of strain within rocks on the northwest ramp of Pine Mountain block, southern Appalachian foreland: A field test of theory. Geol. Soc. Am. Bull. 1985, 96, 426-435. [CrossRef]

21. Kilsdonk, W.; Wiltschko, D.V. Deformation mechanisms in the southeastern ramp region of the Pine Mountain block, Tennessee. Geol. Soc. Am. Bull. 1988, 100, 644-653. [CrossRef]

22. Hnat, J.S.; van der Pluijm, B.A. Foreland signature of indenter tectonics: Insights from calcite twin analysis in the Tennessee salient of the southern Appalachians, USA. Lithosphere 2011, 3, 317-327. [CrossRef]

23. Wiltschko, D.V.; Chapple, W.M. Flow of weak rocks in Appalachian plateau folds. Am. Assoc. Petrol. Geol. 1977, 65, 653-670.

24. Nickelsen, R.P. Fossil distortion and penetrative rock deformation in the Appalachian plateau, Pennsylvania. J. Geol. 1966, 74, 924-931. [CrossRef]

25. Engelder, T.; Engelder, R. Fossil distortion and decollement tectonics of the Appalachian plateau. Geology 1977, 5, 457-460. [CrossRef]

26. Craddock, J.P.; van der Pluijm, B.A. Late Paleozoic deformation of the cratonic carbonate cover of eastern North America. Geology 1989, 17, 416-419. [CrossRef]

27. Craddock, J.P.; Jackson, M.; van der Pluijm, B.A.; Versical, R. Regional shortening fabrics in eastern North America: Far-field stress transmission from the Appalachian-Ouachita orogenic belt. Tectonics 1993, 12, 257-264. [CrossRef]

28. Drake, A.A., Jr. Precambrian and lower Paleozoic geology of the Delaware Valley, New Jersey-Pensylvania. In Geology of Selected Areas in New Jersey and Eastern Pennsylvania; Geological Society of America: Boulder, CO, USA, 1969; pp. 51-205.

29. Drake, A.A., Jr.; Epstein, J.B.; Aaron, J.M. Geologic Maps and Sections of Parts of the Portland and Belvidere Quadrangles; U.S. Geological Survey Miscellaneous Investigations: Easton, PA, USA, 1967.

30. Epstein, J.B. Geology of the Delaware Water Gap National Recreation area, New Jersey-Pennsylvania. Geol. Soc. Am. Field Guides 2006, 8, 47-64.

31. Crawford, M.L.; Mark, L.E. Evidence from metamorphic rocks for overthrusting, Pennsylvania Piedmont, USA. Can. Mineral. 1982, 20, 333-347.

32. Herman, G.C.; Monteverder, D.H.; Schlische, R.W.; Pitcher, D.M. Foreland crustal structure of the New York recess, northeastern United States. Geol. Soc. Am. Bull. 1997, 109, 955-977. [CrossRef]

33. Craddock, J.C. Stratigraphy and Structure of the Kinderhook Quadrangle, New York, and the "Taconic Klippe". Geol. Soc. Am. Bull. 1957, 68, 675-723. [CrossRef]

34. Beutner, E.C.; Diegel, F.A. Determination of fold kinematics from syntectonic fibers in pressure shadows, Martinsburg slate, New Jersey. Am. J. Sci. 1985, 285, 16-50. [CrossRef] 
35. Housen, B.A.; van der Pluijm, B.A. Slaty cleavage development and magneticanisotropy fabrics. J. Geophys. Res. 1991, 96, 9937-9946. [CrossRef]

36. Housen, B.A.; van der Pluijm, B.A.; van Der Voo, R. Magnetite dissolution and neocrystallization during cleavage formation: Paleomagnetic study of the Martinsburg Fm., Lehigh Gap, Pennsylvania. J. Geophys. Res. 1993, 98, 13799-13813. [CrossRef]

37. Pares, J.M.; van der Pluijm, B.A. Magnetic fabrics and strain in pencil structures of the Knobs Fm. Valley and Ridge province, US Appalachians. J. Struct. Geol. 2003, 25, 1349-1358. [CrossRef]

38. Nuriel, P.; Rosenbaum, G.; Zhao, J.-X.; Feng, Y.; Golding, S.D.; Villemant, B.; Weinberger, R. U-Th dating of striated fault planes. Geology 2012, 40, 647-650. [CrossRef]

39. Groshong, R.H., Jr. Strain calculated from twinning in calcite. Bull. Geol. Soc. Am. 1972, 83, $2025-2038$. [CrossRef]

40. Turner, F.J. Nature and dynamic interpretation of deformation lamellae in calcite of three marbles. Am. J. Sci. 1953, 251, 276-298. [CrossRef]

41. Turner, F.J. “Compression" and "tension" axes deduced from $\{0112\}$ twinning in calcite. J. Geophys. Res. 1962, $67,1660$.

42. Paulsen, T.S.; Wilson, T.J.; Demosthenous, C.; Millan, C.; Jarrad, R.; Laufer, A. Kinematics of the Neogene Terror rift: Constraints from calcite twinning strains in the ANDRILL McMurdo Ice Shelf (AND-1B) core, Victoria Land Basin, Antarctica. Geosphere 2014, 10, 828-841. [CrossRef]

43. Craddock, J.P.; van der Pluijm, B. Kinematic analysis of an en echelon-continuous vein complex. J. Struct. Geol. 1988, 10, 445-452. [CrossRef]

44. Engelder, T. The nature of deformation within the outer limits of the central Appalachian foreland fold-and-thrust belt in New York state. Tectonophysics 1979, 55, 289-310. [CrossRef]

45. Spang, J.H.; Groshong, R.H., Jr. Deformation mechanisms and strain history of a minor fold from the Appalachian Valley and Ridge Province. Tectonophysics 1981, 72, 323-342. [CrossRef]

46. Mosar, J. Internal deformation in the Prealpes Medianes, Switzerland. Ecol. Geol. Helv. 1989, 82, 765-793.

47. Ferrill, D.A. Calcite twin widths and intensities as metamorphic indicators in natural low-temperature deformation of limestone. J. Struct. Geol. 1991, 13, 675-677. [CrossRef]

48. Craddock, J.P.; Neilson, K.J.; Malone, D.H. Calcite twinning strain constraints on Heart Mountain detachment kinematics, Wyoming. J. Struct. Geol. 2000, 22, 983-991. [CrossRef]

49. Amrouch, K.; Lacombe, O.; Bellahsen, N.; Daniel, J.M.; Callot, J.P. Stress and strain patterns, kinematics and deformation mechanisms in a basement-cored anticline: Sheep Mountain Anticline, Wyoming. Tectonics 2010, 29. [CrossRef]

50. Craddock, J.P.; Moshoian, A.; Pearson, A.M. Kinematic analysis from twinned calcite strains in the marble mylonites of the central Grenville province, Canada. Geol. Soc. Am. Abstr. Progr. 1991, 23, $236-237$.

51. Craddock, S.D.; Craddock, J.P. Strain Variations in Carbonates across the Proterozoic Grenville Orogen. In Proceedings of the 58th ILSG Annual Meeting, Thunder Bay, ON, Canada, 16-20 May 2012.

52. Craddock, J.P.; Pearson, A. Non-coaxial horizontal shortening strains preserved in amygdule calcite, DSDP Hole 433C, Suiko Seamount. J. Struct. Geol. 1994, 16, 719-724. [CrossRef]

53. Craddock, J.P.; Pearson, A.; McGovern, M.; Kropf, E.P.; Moshoian, A.; Donnelly, K. Post-Extension Shortening Strains Preserved in Calcites of the Keweenawan Rift: Middle Proterozoic to Cambrian Rifting; Ojakgangas, R.W., Dickas, A.B., Green, J.C., Eds.; Geological Society of America Special Paper 213; Geological Society of America: Boulder, CO, USA, 1997.

54. Craddock, J.P.; Farris, D.; Roberson, A. Calcite-twinning constraints on stress-strain fields along the Mid-Atlantic Ridge, Iceland. Geology 2004, 32, 49-52. [CrossRef]

55. Craddock, J.P.; Anziano, J.; Wirth, K.R.; Vervoort, J.D.; Singer, B.; Zhang, X. Structure, geochemistry and geochronology of a lamprophyre dike swarm, Archean Wawa terrane, Michigan, USA. Precambrian Res. 2007, 157, 50-70. [CrossRef]

56. Wenk, H.-R.; Takeshita, T.; Bechler, E.; Erskine, B.G.; Matthies, S. Pure Shear and Simple Shear Calcite Textures. Comparison of Experimental, Theoretical and Natural Data. J. Struct. Geol. 1987, 9, 731-745. [CrossRef]

57. Burkhard, M. Calcite twins, their geometry, appearance and significance as stress-strain markers and indicators of tectonic regime: A review. J. Struct. Geol. 1993, 15, 351-368. [CrossRef] 
58. Lacombe, O.; Laurent, P. Determination of deviatoric stress tensors based on inversion of calcite twin data from experimentally deformed monophase samples: Preliminary results. Tectonophysics 1996, 255, 189-202. [CrossRef]

59. Ferrill, D.A. Critical re-evaluation of differential stress estimates from calcite twins in coarse-grained limestone. Tectonophysics 1998, 285, 77-86. [CrossRef]

60. Groshong, R.H., Jr.; Teufel, L.W.; Gasteiger, C.M. Precision and accuracy of the calcite strain-gage technique. Bull. Geol. Soc. Am. 1984, 95, 357-363. [CrossRef]

61. Groshong, R.H., Jr. Experimental test of least-squares strain calculations using twinned calcite. Bull. Geol. Soc. Am. 1974, 85, 1855-1864. [CrossRef]

62. Jamison, W.R.; Spang, J.H. Use of calcite twin lamellae to infer differential stress. Bull. Geol. Soc. Am. 1976, 87, 868-872. [CrossRef]

63. Rowe, K.J.; Rutter, E.H. Paleostress estimation using calcite twinning: Experimental calibration and application to nature. J. Struct. Geol. 1990, 12, 1-17. [CrossRef]

64. Teufel, L.W. Strain analysis of experimental superposed deformation using calcite twin lamellae. Tectonophysics 1980, 65, 291-309. [CrossRef]

65. Evans, M.A.; Groshong, R.H., Jr. A Computer Program for the Calcite Strain-Gage Technique. J. Struct. Geol. 1994, 16, 277-281. [CrossRef]

66. Ferrill, D.A.; Morris, A.P.; Evans, M.A.; Burkhard, M.; Groshong, R.H.; Onasch, C.M. Calcite Twin Morphology: A Low-Temperature Deformation Geothermometer. J. Struct. Geol. 2004, 26, 1521-1529. [CrossRef]

67. Gray, M.B.; Stamatakos, J.A.; Ferrill, D.A.; Evans, M.A. Fault-Zone Deformation in Welded Tuffs at Yucca Mountain, Nevada, USA. J. Struct. Geol. 2005, 27, 1873-1891. [CrossRef]

68. Lohmann, K.C.; (University of Michigan, Ann Arbor, MI, USA). Personal communication, 2012.

69. Craddock, J.P.; Konstantinou, A.; Vervoort, J.D.; Wirth, K.R.; Davidson, C.; Finley-Blasi, L.; Juda, N.A.; Walker, E. Detrital Zircon Provenance of the Proterozoic Midcontinent Rift, Lake Superior region, USA. J. Geol. 2013, 121, 57-73. [CrossRef]

70. Passchier, C.W.; Trouw, R.A.J. Microtectonics; ISBN: 978-3-540-64003-5. Springer: Berlin, Germany, 2005.

71. Craddock, J.P. Transpression during tectonic evolution of the Idaho-Wyoming fold-and-thrust belt. In Regional Geology of Eastern Idaho and Western Wyoming, Edition: Memoir 179, Chapter: 6; Link, P.K., Ed.; Geologic Society of America: Boulder, CO, USA, 1992; pp. 125-139.

72. Van Staal, C.; Dewey, J.F.; Mac Niocaill, C.; McKerrow, W.S. The Cambrian-Silurian tectonic evolution of the northern Appalachians and British Caledonides: History of a complex, west and southwest Pacific-type segment of Iapetus: Geological Society, London. Spec. Publ. 1998, 143, 197-242. [CrossRef]

73. Freedman, J.; Bentley, R.; Wise, D. Pattern of folded folds in the Appalachian Piedmont along Susquehanna River. Geol. Soc. Am. Bull. 1964, 75, 621-638. [CrossRef]

74. Faill, R.T. The geologic history of the north-central Appalachians; Part 3, The Alleghany Orogeny. Am. J. Sci. 1998, 298, 131-179. [CrossRef]

75. Groshong, R.H., Jr. Strain, fractures, and pressure solution in natural single-layer. Bull. Geol. Soc. Am. 1975, 86, 1363-1376. [CrossRef]

76. Wise, D.U.; Werner, M.L. Pennsylvania salient of the Appalachians: New Piedmont data suggest origin by two movement directions on a regional decollement. In Orogenic Curvature: Geological Society of America Special Paper 383; Sussman, A.J., Weil, A.B., Eds.; Geological Society of America: Boulder, CO, USA, 2004; pp. 109-120.

77. Miller, J.A.; Kent, D.V. Regional trends in the timing of Alleghenian remagnetization in the Appalachians. Geology 1988, 16, 195-198. [CrossRef]

78. Schwartz, S.Y; van der Voo, R. Paleomagnetic evaluation of the orocline hypothesis in the central and southern Appalachians. Geophys. Res. Lett. 1983, 10, 505-508. [CrossRef]

79. Stamatakos, J.; Hirt, A.M. Paleomagnetic considerations of the development of the Pennsylvania salient in the central Appalachians. Tectonophysics 1994, 231, 237-255. [CrossRef]

80. Stamatakos, J.; Hirt, A.M.; Lowrie, W. The age and timing of folding in the central Appalachians from paleomagnetic results. Geol. Soc. Am. Bull. 1996, 108, 815-829. [CrossRef]

81. Gray, M.B.; Stamatakos, J. New model for evolution of fold and thrust belt curvature based on integrated structural and paleomagnetic results from the Pennsylvania salient. Geology 1997, 25, 1067-1070. [CrossRef] 
82. Elmore, R.D.; Kelley, J.; Evans, M.; Lewchuk, M.T. Remagnetization and orogenic fluids: Testing the hypothesis in the central Appalachians. Geophys. J. Int. 2001, 144, 568-576. [CrossRef]

83. Evans, M.A.; Elmore, R.D.; Lewchuk, M.T. Examining the relationship between remagnetization and orogenic fluids: Central Appalachians. J. Geochem. Explor. 2001, 69, 139-142. [CrossRef]

84. Hnat, J.S.; van der Pluijm, B.A.; van der Voo, R.; Thomas, W.A. Differential displacement and rotation in thrust fronts: A magnetic, calcite twinning and palinspastic study of the Jones Valley thrust, Alabama, US Appalachians. J. Struct. Geol. 2008, 30, 725-738. [CrossRef]

85. Hnat, J.S.; van der Pluijm, B.A.; van der Voo, R. Remagnetization in the Tennessee salient, Southern Appalachians, USA: Constraints on the timing of deformation. Tectonophysics 2009, 474, 709-722. [CrossRef]

86. Geiser, P.A. Slaty cleavage and the dewatering hypothesis-An examination of some critical evidence. Geology 1975, 3, 717-721. [CrossRef]

87. Valentino, D.W.; Gates, A.E. The Mid-Atlantic Piedmont: Tectonic Missing Link of the Appalachians; Geological Society of America Special Paper 330; Geological Society of America: Boulder, CO, USA, 1999; p. 139.

88. Lomando, A.J.; Engelder, T. Strain indicated by calcite twinning: Implications for deformation of the early Mesozoic northern Newark Basin, New York. Northeast Geol. 1984, 6, 192-195.

89. Anderson, E.M. The Dynamics of Faulting, 2nd ed.; Oliver \& Boyd: Edinburgh, UK, 1951; p. 208.

90. Craddock, J.P.; Klein, T.; Kowalczyk, G.; Zulauf, G. Calcite twinning strains in Alpine orogen flysch: Implications for thrust-nappe mechanics and the geodynamics of Crete. Lithosphere 2009, 1, 174-191. [CrossRef]

(C) 2016 by the authors; licensee MDPI, Basel, Switzerland. This article is an open access article distributed under the terms and conditions of the Creative Commons by Attribution (CC-BY) license (http:/ / creativecommons.org/licenses/by/4.0/). 